\title{
Variational Problems with Partial Fractional Derivative: Optimal Conditions and Noether's Theorem
}

\author{
Jun Jiang, ${ }^{1,2}$ Yuqiang Feng $\mathbb{D}^{1,2}$ and Shougui $\mathrm{Li}^{1,2}$ \\ ${ }^{1}$ School of Science, Wuhan University of Science and Technology, Wuhan 430065, Hubei, China \\ ${ }^{2}$ Hubei Province Key Laboratory of Systems Science in Metallurgical Process, Wuhan 430065, Hubei, China \\ Correspondence should be addressed to Yuqiang Feng; yqfeng6@126.com
}

Received 8 June 2018; Accepted 10 September 2018; Published 2 October 2018

Academic Editor: Henryk Hudzik

Copyright (c) 2018 Jun Jiang et al. This is an open access article distributed under the Creative Commons Attribution License, which permits unrestricted use, distribution, and reproduction in any medium, provided the original work is properly cited.

In this paper, the necessary and sufficient conditions of optimality for variational problems with Caputo partial fractional derivative are established. Fractional Euler-Lagrange equations are obtained. The Legendre condition and Noether's theorem are also presented.

\section{Introduction}

Since the introduction of fractional calculus of variations by Riewe [1], fractional calculus has been a subject of interest not only among mathematicians, but also among fluid mechanics, electricity and finance specialists, chemical physicists, biomedical engineering specialists, and control theory specialists.

Considerable progress has been made to determine necessary and sufficient conditions that any extremal for the variational functional with fractional calculus must satisfy in recent years. R. Almeida [2] provides necessary and sufficient conditions of optimality for variational problems that deal with a fractional derivative with respect to another function. Almeida established the fractional Euler-Lagrange equations for the fundamental problem and when in presence of an integral constraint and Almeida obtained a Legendre condition. In [3] Almeida studied certain problems of calculus of variations that are dependent upon a Lagrange function on a Caputo-type fractional derivative; sufficient and necessary conditions of the first- and second-order are presented. In [4] Zhang Jianke, Ma Xiaojue, and Li Lifeng studied the necessary and sufficient optimality conditions for problems of the fractional calculus of variations with a Lagrange function depending on a Caputo-Fabrizio fractional derivative. In [5] Almeida et al. obtained necessary optimality conditions for variational problems with a Lagrangian depending on a Caputo fractional derivative and indefinite integral. There has been a significant development in ordinary and partial fractional differential equations in recent years [6-9].

D. Tavares et al. in [10] presented two fractional isoperimetric problems where the Lagrangian depends on a combined Caputo derivative of variable fractional order and presented a new variational problem subject to holonomic constraint.

Noether's symmetry, namely, the invariance of Hamilton action under the infinitesimal transformations, is put forward for the first time by Noether [11]. In [12] Frederico et al. obtained a generalization of the Noether theorem for Lagrangians depending on mixed classical and Caputo derivatives that can be used to obtain constants of motion for dissipative systems. In [13] the Noether theorem and its inverse theorem for the nonlinear dynamical systems with nonstandard Lagrangians are studied. In [14] a variational principle for Lagrangian densities containing derivatives of real order was formulated and the invariance of this principle is studied in two characteristic cases. In [15] Yan B. et al. studied Noether's symmetries and conserved quantities of the Birkhoffian systems in terms of fractional derivatives of variable order.

In this paper, we extend the study to variational problems involving partial fractional derivatives. Our aim is to obtain 
the necessary and sufficient conditions for the minimizer. Moreover, we will establish Noether's theorem for these problems.

The paper is organized as follows. In Section 2, the basic definitions and notations are given. In Sections 3.1 and 3.2, the first-order necessary condition and the second-order necessary condition for the minimizer are established. Then, the variational problem subject to an integral constraint is investigated in Section 3.3 and the isoperimetric problem is discussed in Section 3.4. In Section 3.5, Noether's theorem for this system is proved. In Section 4, as applications of our results, some examples are presented.

\section{Preliminaries}

In this section, we will recall some basic concepts and preliminary results on fractional calculus, needed in the sequel. To fix notation, in the following $D=[a, b] \times[c, d]$ and $\partial D$ is the boundary of $D$.

Definition 1 (Riemann-Liouville fractional integrals, [12]). The left and right Riemann-Liouville fractional integrals of order $\alpha$ for the function $f(x)$ are defined, respectively, as

$$
\begin{aligned}
& I_{a+}^{\alpha} f(t):=\frac{1}{\Gamma(\alpha)} \int_{a}^{t}(t-\tau)^{\alpha-1} f(\tau) d \tau \\
& I_{b-}^{\alpha} f(t):=\frac{1}{\Gamma(\alpha)} \int_{t}^{b}(\tau-t)^{\alpha-1} f(\tau) d \tau
\end{aligned}
$$

where $\Gamma$ is the gamma function.

Definition 2 (Riemann-Liouville fractional derivatives, [12]). The left and right Riemann-Liouville fractional derivatives of order $\alpha$ for the function $f(x)$ are defined, respectively, as

$$
\begin{aligned}
& D_{a+}^{\alpha} f(t):=\frac{1}{\Gamma(1-\alpha)} \frac{d}{d t} \int_{a}^{t}(t-\tau)^{-\alpha} f(\tau) d \tau \\
& D_{b-}^{\alpha} f(t):=\frac{1}{\Gamma(1-\alpha)} \frac{d}{d t} \int_{t}^{b}(\tau-t)^{-\alpha} f(\tau) d \tau
\end{aligned}
$$

Remark 3. The Riemann-Liouville fractional derivative of a constant $c$ need not to be zero.

Definition 4 (Caputo fractional derivatives, [12]). The left Caputo fractional derivative of $f(x)$ of order $\alpha \in(0,1)$ is defined by

$$
\begin{aligned}
& { }^{C} D_{a+}^{\alpha} f(t) \\
& \quad:=\frac{1}{\Gamma(1-\alpha)} \frac{d}{d t} \int_{a}^{t} \frac{1}{(t-\tau)^{\alpha}}[f(\tau)-f(a)] d \tau
\end{aligned}
$$

If $f$ is of class $C^{1}$, then we have the equivalent form

$$
{ }^{C} D_{a+}^{\alpha} f(t):=\frac{1}{\Gamma(1-\alpha)} \int_{a}^{t} \frac{1}{(t-\tau)^{\alpha}} f^{\prime}(\tau) d \tau
$$

while the right Caputo fractional derivative of $f(x)$ of order $\alpha \in(0,1)$ is given by

$$
{ }^{C} D_{b-}^{\alpha} f(t):=\frac{1}{\Gamma(1-\alpha)} \int_{t}^{b} \frac{1}{(\tau-t)^{\alpha}} f^{\prime}(\tau) d \tau
$$

Remark 5. The Caputo derivative exhibits an important feature: the derivative of a constant is zero.

Definition 6 (partial Riemann-Liouville integrals, [9]). Let $\alpha \in(0,1)$ and $u \in L^{1}(D)$. The left and right partial RiemannLiouville integrals of order $\alpha \in(0,1)$ of $u(x, y)$ with respect to $x$ are defined, respectively, by the expression

$$
\begin{aligned}
& I_{a, x}^{\alpha} u(x, y)=\frac{1}{\Gamma(\alpha)} \int_{a}^{x}(x-s)^{\alpha-1} u(s, y) d s \\
& I_{x, b}^{\alpha} u(x, y)=\frac{1}{\Gamma(\alpha)} \int_{x}^{b}(s-x)^{\alpha-1} u(s, y) d s
\end{aligned}
$$

for almost all $(x, y) \in D$. Analogously, we define the integrals

$$
\begin{aligned}
& I_{c, y}^{\alpha} u(x, y)=\frac{1}{\Gamma(\alpha)} \int_{c}^{y}(y-t)^{\alpha-1} u(x, t) d t \\
& I_{y, d}^{\alpha} u(x, y)=\frac{1}{\Gamma(\alpha)} \int_{y}^{d}(t-y)^{\alpha-1} u(x, t) d t
\end{aligned}
$$

for almost all $(x, y) \in D$.

Definition 7 (partial Riemann-Liouville derivatives, [9]). Let $\alpha \in(0,1)$ and $u \in L^{1}(D)$. The left and right partial RiemannLiouville derivatives of order $\alpha \in(0,1)$ of $u(x, y)$ with respect to $x$ are defined, respectively, by the expression

$$
\begin{aligned}
& D_{a, x}^{\alpha} u(x, y)=\frac{\partial}{\partial x} I_{a, x}^{1-\alpha} u(x, y) \\
& D_{x, b}^{\alpha} u(x, y)=\frac{\partial}{\partial x} I_{x, b}^{1-\alpha} u(x, y)
\end{aligned}
$$

for almost all $(x, y) \in D$. Analogously, we define the derivatives

$$
\begin{aligned}
& D_{c, y}^{\alpha} u(x, y)=\frac{\partial}{\partial y} I_{c, y}^{1-\alpha} u(x, y) \\
& D_{y, d}^{\alpha} u(x, y)=\frac{\partial}{\partial y} I_{y, d}^{1-\alpha} u(x, y)
\end{aligned}
$$

for almost all $(x, y) \in D$.

Definition 8 (partial Caputo fractional derivative, [9]). Let $\alpha \in(0,1)$ and $u \in L^{1}(D)$. The left and right partial Caputo fractional derivatives of order $\alpha \in(0,1)$ of $u(x, y)$ with respect to $x$ are defined, respectively, by the expression

$$
\begin{aligned}
& { }^{C} D_{a, x}^{\alpha} u(x, y)=I_{a, x}^{1-\alpha} \frac{\partial}{\partial x} u(x, y) \\
& { }^{C} D_{x, b}^{\alpha} u(x, y)=I_{x, b}^{1-\alpha} \frac{\partial}{\partial x} u(x, y)
\end{aligned}
$$


for almost all $(x, y) \in D$. Analogously, we define the derivatives

$$
\begin{aligned}
& { }^{C} D_{c, y}^{\alpha} u(x, y)=I_{c, y}^{1-\alpha} \frac{\partial}{\partial y} u(x, y) \\
& { }^{C} D_{x, b}^{\alpha} u(x, y)=I_{x, b}^{1-\alpha} \frac{\partial}{\partial x} u(x, y)
\end{aligned}
$$

for almost all $(x, y) \in D$.

Theorem 9. Let $f(x, y)$ be a continuous function and $g(x, y)$ be of class $C^{1}$; then

$$
\begin{aligned}
\iint_{D} f(x, y) \cdot{ }^{C} D_{a, x}^{\alpha} g(x, y) d x d y \\
=\iint_{D} \frac{\partial}{\partial x}\left(g(x, y) \cdot I_{x, b}^{1-\alpha} f(x, y)\right) d x d y \\
\quad-\iint_{D}\left(g(x, y) \cdot D_{x, b}^{\alpha} f(x, y)\right) d x d y
\end{aligned}
$$

and

$$
\begin{aligned}
\iint_{D} f(x, y) \cdot{ }^{C} D_{c, y}^{\alpha} g(x, y) d x d y \\
=\iint_{D} \frac{\partial}{\partial y}\left(g(x, y) \cdot I_{y, d}^{1-\alpha} f(x, y)\right) d x d y \\
\quad-\iint_{D}\left(g(x, y) \cdot D_{y, d}^{\alpha} f(x, y)\right) d x d y
\end{aligned}
$$

Proof.

$$
\begin{aligned}
& \iint_{D} f(x, y) \cdot{ }^{C} D_{a, x}^{\alpha} g(x, y) d x d y=\int_{c}^{d} \int_{a}^{b}(f(x, y) \\
& \left.\cdot \frac{1}{\Gamma(1-\alpha)} \int_{a}^{x}(x-s)^{-\alpha} \frac{\partial}{\partial s} g(s, y) d s\right) d x d y \\
& =\int_{c}^{d}\left(\int_{a}^{b} f(x, y)\right. \\
& \left.\cdot \frac{1}{\Gamma(1-\alpha)} \int_{a}^{x}(x-s)^{-\alpha} \frac{\partial}{\partial s} g(s, y) d s d x\right) d y \\
& =\int_{c}^{d}\left(\int_{a}^{b} \frac{\partial}{\partial s} g(s, y)\right. \\
& \left.\cdot \frac{1}{\Gamma(1-\alpha)} \int_{s}^{b}(x-s)^{-\alpha} f(x, y) d x d s\right) d y \\
& =\int_{c}^{d}\left(\int_{a}^{b} \frac{\partial}{\partial x} g(x, y)\right. \\
& \left.\cdot \frac{1}{\Gamma(1-\alpha)} \int_{x}^{b}(s-x)^{-\alpha} f(s, y) d s d x\right) d y
\end{aligned}
$$

$$
\begin{aligned}
& =\int_{c}^{d} \int_{a}^{b}\left(\frac{\partial}{\partial x} g(x, y) \cdot \frac{1}{\Gamma(1-\alpha)}\right. \\
& \left.\cdot \int_{x}^{b}(s-x)^{-\alpha} f(s, y) d s\right) d x d y \\
& =\iint_{D} \frac{\partial}{\partial x}\left(\frac{g(x, y)}{\Gamma(1-\alpha)} \int_{x}^{b}(s-x)^{-\alpha}\right. \\
& \cdot f(s, y) d s) d x d y \\
& -\iint_{D}\left(\frac{g(x, y)}{\Gamma(1-\alpha)} \frac{\partial}{\partial x} \int_{x}^{b}(s-x)^{-\alpha}\right. \\
& \cdot f(s, y) d s) d x d y \\
& =\iint_{D} \frac{\partial}{\partial x}\left(g(x, y) \cdot I_{x, b}^{1-\alpha} f(x, y)\right) d x d y \\
& -\iint_{D}\left(g(x, y) \cdot D_{x, b}^{\alpha} f(x, y)\right) d x d y
\end{aligned}
$$

Analogously, we obtain

$$
\begin{aligned}
\iint_{D} f(x, y) \cdot{ }^{C} D_{c, y}^{\alpha} g(x, y) d x d y \\
=\iint_{D} \frac{\partial}{\partial y}\left(g(x, y) \cdot I_{y, d}^{1-\alpha} f(x, y)\right) d x d y \\
\quad-\iint_{D}\left(g(x, y) \cdot D_{y, d}^{\alpha} f(x, y)\right) d x d y
\end{aligned}
$$

Theorem 10 (see [16]). If $f(x, y) \in C^{1}(D)$ satisfies

$$
\iint_{D} f(x, y) \eta(x, y) d x d y=0
$$

for all $\eta(x, y) \in C^{1}(D)$ with $\left.\eta(x, y)\right|_{\partial D}=0$, then $f(x, y) \equiv 0$ on $D$.

\section{The Variational Problem}

The aim of this section is to study problems of fractional calculus of variations, where the integral functional depends on the partial Caputo fractional derivative. Given $u(x, y) \in$ $C^{1}(D)$, we define the functional

$$
\begin{gathered}
J(u(x, y))=\iint_{D} F\left(x, y, u(x, y), u_{x}(x, y), u_{y}(x, y),\right. \\
\left.{ }^{C} D_{a, x}^{\alpha} u(x, y),{ }^{C} D_{c, y}^{\alpha} u(x, y)\right) d x d y
\end{gathered}
$$

with the following assumptions:

(i) $F: D \times R^{5} \longrightarrow R$ is a continuous function, such that $\partial_{3} F, \partial_{4} F, \partial_{5} F, \partial_{6} F$, and $\partial_{7} F$ exist and are continuous. 
(ii) Given any $u(x, y) \in C^{1}(D),{ }^{C} D_{x, b}^{\alpha} \partial_{6} F$ and ${ }^{C} D_{y, d}^{\alpha} \partial_{7} F$ are continuous.

For simplification, we consider the operator

$$
\begin{gathered}
{[u](x, y)=\left(x, y, u(x, y), u_{x}(x, y), u_{y}(x, y)\right.} \\
\left.{ }^{C} D_{a, x}^{\alpha} u(x, y),{ }^{C} D_{c, y}^{\alpha} u(x, y)\right) \\
\partial_{i} h\left(x_{1}, x_{2}, \ldots, x_{n}\right)=\frac{\partial h}{\partial x_{i}}\left(x_{1}, x_{2}, \ldots, x_{n}\right) \\
\partial_{i j}^{2} h\left(x_{1}, x_{2}, \ldots, x_{n}\right)=\frac{\partial^{2} h}{\partial x_{i} \partial x_{j}}\left(x_{1}, x_{2}, \ldots, x_{n}\right)
\end{gathered}
$$

\subsection{The Fundamental Problem}

Theorem 11. Suppose that $u^{*}(x, y)$ is a local minimizer for $J$ as in (17), defined on

$$
\begin{aligned}
U & =\left\{u(x, y) \in C^{1}(D) \mid u(x, c)=\varphi(x), u(a, y)\right. \\
& =\psi(y), x \in[a, b], y \in[c, d]\}
\end{aligned}
$$

where $\varphi(x) \in A C([a, b])$ and $\psi(y) \in A C([c, d])$. Then $u^{*}(x, y)$ is a solution to the equation

$$
\begin{aligned}
\partial_{3} F\left[u^{*}\right](x, y)-\frac{\partial}{\partial x}\left(\partial_{4} F\left[u^{*}\right](x, y)\right) \\
-\frac{\partial}{\partial y} \partial_{5} F\left[u^{*}\right](x, y)-D_{x, b}^{\alpha} \partial_{6} F\left[u^{*}\right](x, y) \\
-D_{y, d}^{\alpha} \partial_{7} F\left[u^{*}\right](x, y)=0
\end{aligned}
$$

for almost all $(x, y) \in D$.

Proof. Let $u(x, y)+\varepsilon \eta(x, y)$ be a variation of $u(x, y)$, with $|\varepsilon| \ll 1, \eta(x, y) \in C^{1}(D) \cap U$, and $\left.\eta(x, y)\right|_{\partial D}=0$. We define the function $h$ in a neighborhood of zero by the expression

$$
h(\varepsilon)=J\left(u^{*}+\varepsilon \eta\right)
$$

Since $u^{*}$ is a minimizer of $J$, then $\varepsilon=0$ is a minimizer of $h$, and so $h^{\prime}(0)=0$. Differentiating $h$ at $\varepsilon=0$ and using Theorem 9, we obtain

$$
\begin{aligned}
& h^{\prime}(0)=\iint_{D}\left(\partial_{3} F\left[u^{*}\right](x, y) \cdot \eta(x, y)+\partial_{4} F\left[u^{*}\right]\right. \\
& \cdot(x, y) \cdot \partial_{x} \eta(x, y)+\partial_{5} F\left[u^{*}\right](x, y) \cdot \partial_{y} \eta(x, y) \\
& \quad+\partial_{6} F\left[u^{*}\right](x, y) \cdot{ }^{C} D_{a, x}^{\alpha} \eta(x, y)+\partial_{7} F\left[u^{*}\right](x, y) \\
& \left.\quad{ }^{C} D_{c, y}^{\alpha} \eta(x, y)\right) d x d y \Longrightarrow
\end{aligned}
$$

$$
\begin{aligned}
& h^{\prime}(0)=\iint_{D}\left(\partial_{3} F\left[u^{*}\right](x, y)-\frac{\partial}{\partial x}\left(\partial_{4} F\left[u^{*}\right](x, y)\right)\right. \\
& -\frac{\partial}{\partial y} \partial_{5} F\left[u^{*}\right](x, y)-D_{x, b}^{\alpha} \partial_{6} F\left[u^{*}\right](x, y) \\
& \left.-D_{y, d}^{\alpha} \partial_{7} F\left[u^{*}\right](x, y)\right) \eta(x, y) d x d y \\
& +\iint_{D} \frac{\partial}{\partial x}\left(\left(\partial_{4} F\left[u^{*}\right](x, y)+I_{x, b}^{1-\alpha} \partial_{6} F\left[u^{*}\right](x, y)\right)\right. \\
& \cdot \eta(x, y)) d x d y \\
& +\iint_{D} \frac{\partial}{\partial y}\left(\left(\partial_{5} F\left[u^{*}\right](x, y)+I_{y, d}^{1-\alpha} \partial_{7} F\left[u^{*}\right](x, y)\right)\right. \\
& \cdot \eta(x, y)) d x d y \Longrightarrow \\
& h^{\prime}(0)=0 . \\
& \quad-(x, y)) \eta(x, y) d y \Longrightarrow \\
& \quad-D_{\partial D}\left(\partial_{5} F\left[u^{*}\right](x, y)+I_{y, d}^{1-\alpha} \partial_{7} F\left[u^{*} F\left[u^{*}\right](x, y)\right) \eta(x, \eta(x, y) d x d y\right. \\
& \quad-\frac{\partial}{\partial y} \partial_{5} F\left[u^{*}\right](x, y)-D_{x, b}^{\alpha} \partial_{6} F\left[u^{*}\right](x, y) \\
& h^{\prime}(x, y)-\frac{\partial}{\partial x}\left(\partial_{4} F\left[u^{*}\right](x, y)\right)
\end{aligned}
$$

Since $\left.\eta(x, y)\right|_{\partial D}=0$ and $\eta(x, y)$ is arbitrary elsewhere, we conclude that

$$
\begin{aligned}
\partial_{3} F\left[u^{*}\right](x, y)-\frac{\partial}{\partial x}\left(\partial_{4} F\left[u^{*}\right](x, y)\right) \\
-\frac{\partial}{\partial y} \partial_{5} F\left[u^{*}\right](x, y)-D_{x, b}^{\alpha} \partial_{6} F\left[u^{*}\right](x, y) \\
-D_{y, d}^{\alpha} \partial_{7} F\left[u^{*}\right](x, y)=0
\end{aligned}
$$

for almost all $(x, y) \in D$.

Remark 12. Equation (20) is called the Euler-Lagrange equation associated with the functional $J$. Solutions of this equation are called extremals.

Remark 13. If we define functional

$$
\begin{gathered}
J(u(x, y))=\iint_{D} F\left(x, y, u(x, y),{ }^{C} D_{a, x}^{\alpha} u(x, y),\right. \\
\left.{ }^{C} D_{c, y}^{\alpha} u(x, y)\right) d x d y
\end{gathered}
$$


then we obtain the Euler-Lagrange equation

$$
\begin{gathered}
\partial_{3} F\left[u^{*}\right](x, y)-D_{x, b}^{\alpha} \partial_{4} F\left[u^{*}\right](x, y) \\
-D_{y, d}^{\alpha} \partial_{5} F\left[u^{*}\right](x, y)=0
\end{gathered}
$$

for almost all $(x, y) \in D$.

3.2. The Legendre Condition. In [17], a second-order necessary condition had been proved for functionals involving Riemann-Liouville fractional derivatives.

In this section, we give a second-order necessary condition, usually called Legendre condition, for functionals involving Riemann-Liouville partial fractional derivatives.

We introduce the functional

$$
\begin{aligned}
& J(u(x, y))=\iint_{D} F\left(x, y, u(x, y),{ }^{C} D_{a, x}^{\alpha} u(x, y),\right. \\
& \left.{ }^{C} D_{c, y}^{\alpha} u(x, y)\right) d x d y
\end{aligned}
$$

with the same assumptions on $F$ as in Section 3.1.

Theorem 14. Suppose that ${ }^{*}$ is a local minimizer for J in U. If $\partial_{i j}^{2} F$ exists and is continuous for $i, j \in\{3,4,5\}$, then $u^{*}$ satisfies

$$
\begin{aligned}
& \partial_{44} F\left[u^{*}\right](x, y) \geq 0 \\
& \partial_{55} F\left[u^{*}\right](x, y) \geq 0
\end{aligned}
$$

for almost all $(x, y) \in D$.

Proof. Let $u^{*}(x, y)+\varepsilon \eta(x, y)$ be a variation of $u^{*}(x, y)$, with $|\varepsilon| \ll 1, \eta(x, y) \in C^{1}(D) \cap U$, and $\left.\eta(x, y)\right|_{\partial D}=0$.

We define the function $h$ in a neighborhood of zero by the expression $h(\varepsilon)=J\left(u^{*}+\varepsilon \eta\right)$. Then, we have $h^{\prime \prime}(0) \geq 0$; that is,

$$
\begin{aligned}
& \iint_{D}\left(\partial_{33} F\left[u^{*}\right](x, y) \cdot \eta^{2}(x, y)+2 \partial_{34} F\left[u^{*}\right](x, y)\right. \\
& \cdot \eta(x, y) \cdot{ }^{C} D_{a, x}^{\alpha} \eta(x, y)+2 \partial_{35} F\left[u^{*}\right](x, y) \\
& \cdot \eta(x, y) \cdot{ }^{C} D_{c, y}^{\alpha} \eta(x, y)+\partial_{44} F\left[u^{*}\right](x, y) \\
& \cdot\left({ }^{C} D_{a, x}^{\alpha} \eta(x, y)\right)^{2}+2 \partial_{45} F\left[u^{*}\right](x, y) \\
& \cdot{ }^{C} D_{a, x}^{\alpha} \eta(x, y) \cdot{ }^{C} D_{c, y}^{\alpha} \eta(x, y)+\partial_{55} F\left[u^{*}\right](x, y) \\
& \left.\cdot\left({ }^{C} D_{c, y}^{\alpha} \eta(x, y)\right)^{2}\right) d x d y \geq 0
\end{aligned}
$$

Assume that the Legendre condition is violated at some $\left(x_{0}, y_{0}\right) \in D$; i.e.,

$$
\partial_{55} F\left[u^{*}\right](x, y)<0
$$

Then, there exists a rectangle $[\bar{a}, \bar{b}] \times[\bar{c}, \bar{d}] \triangleq \bar{D} \in D$ and six real constants $C_{1}, C_{2}, C_{3}, C_{4}, C_{5}, C_{6}$ with $C_{6}<0$, such that

$$
\begin{aligned}
& \partial_{33} F\left[u^{*}\right](x, y) \leq C_{1}, \\
& \partial_{34} F\left[u^{*}\right](x, y) \leq C_{2}, \\
& \partial_{35} F\left[u^{*}\right](x, y) \leq C_{3}, \\
& \partial_{44} F\left[u^{*}\right](x, y) \leq C_{4}, \\
& \partial_{45} F\left[u^{*}\right](x, y) \leq C_{5}, \\
& \partial_{55} F\left[u^{*}\right](x, y) \leq C_{6}<0,
\end{aligned}
$$

for all $(x, y) \in \bar{D}$.

Define the function $h(x, y):[\bar{a}, \bar{b}] \times[\bar{c}, \bar{d}] \longrightarrow R$ as follows:

$$
\begin{array}{r}
h(s, t)=\left((\alpha+2)(s-\bar{a})^{1+\alpha}-2 \frac{\alpha+4}{\bar{b}-\bar{a}}(s-\bar{a})^{2+\alpha}\right. \\
\left.+\frac{\alpha+10}{(\bar{b}-\bar{a})^{2}}(s-\bar{a})^{3+\alpha}-\frac{4}{(\bar{b}-\bar{a})^{3}}(s-\bar{a})^{4+\alpha}\right) \\
\cdot\left((\alpha+2)(t-\bar{c})^{1+\alpha}-2 \frac{\alpha+4}{\bar{d}-\bar{c}}(t-\bar{c})^{2+\alpha}\right. \\
\left.+\frac{\alpha+10}{(\bar{d}-\bar{c})^{2}}(t-\bar{c})^{3+\alpha}-\frac{4}{(\bar{d}-\bar{c})^{3}}(t-\bar{c})^{4+\alpha}\right)
\end{array}
$$

Then, $h(s, t)$ is of class $C^{1}$, and

$$
\begin{aligned}
h(\bar{a}, t) & =0=h(\bar{b}, t), \quad t \in[\bar{c}, \bar{d}] \\
h(s, \bar{c})=0 & =h(s, \bar{d}), \quad s \in[\bar{a}, \bar{b}] \\
h_{s}^{\prime}(\bar{a}, t)=0 & =h_{s}^{\prime}(\bar{b}, t), \quad t \in[\bar{c}, \bar{d}] \\
h_{t}^{\prime}(s, \bar{c})=0 & =h_{t}^{\prime}(s, \bar{d}), \quad s \in[\bar{a}, \bar{b}] \\
{ }^{C} D_{\bar{a}, x}^{\alpha} h(\bar{a}, t)=0 & ={ }^{C} D_{\bar{a}, x}^{\alpha} h(\bar{b}, t), \quad t \in[\bar{c}, \bar{d}] \\
{ }^{C} D_{\bar{c}, y}^{\alpha} h(s, \bar{c})=0 & ={ }^{C} D_{\bar{c}, y}^{\alpha} h(s, \bar{d}), \quad s \in[\bar{a}, \bar{b}]
\end{aligned}
$$

Moreover, for every $(x, y) \in \bar{D}$,

$$
\begin{aligned}
h(x, y) & \leq(14)^{2}(\bar{b}-\bar{a})^{1+\alpha}(\bar{d}-\bar{c})^{1+\alpha} \\
{ }^{C} D_{\bar{a}, x}^{\alpha} h(x, y) & \leq \bar{C}(\bar{b}-\bar{a})(\bar{d}-\bar{c})^{1+\alpha}
\end{aligned}
$$

and

$$
{ }^{C} D_{\bar{c}, y}^{\alpha} h(x, y) \leq \bar{C}(\bar{b}-\bar{a})^{1+\alpha}(\bar{d}-\bar{c}), \quad \bar{C}=700
$$

Let

$$
\bar{\eta}(x, y)= \begin{cases}h(x, y), & (x, y) \in \bar{D} \\ 0, & \text { otherwise }\end{cases}
$$


By the properties of function $h(x, y)$, we have $\bar{\eta}(x, y) \in$ $C^{1}(D),\left.\bar{\eta}\right|_{\partial D}=0$, and

$$
\begin{aligned}
& { }^{C} D_{a, x}^{\alpha} \bar{\eta}(x, y)= \begin{cases}{ }^{C} D_{\bar{a}, x}^{\alpha} h(x, y), & (x, y) \in \bar{D} \\
0, & \text { otherwise }\end{cases} \\
& { }^{C} D_{c, y}^{\alpha} \bar{\eta}(x, y)= \begin{cases}{ }^{C} D_{\bar{c}, y}^{\alpha} h(x, y), & (x, y) \in \bar{D} \\
0, & \text { otherwise }\end{cases}
\end{aligned}
$$

Bringing this variation into (28), we get

$$
\begin{aligned}
& 0 \leq \iint_{D}\left(\partial_{33} F\left[u^{*}\right](x, y) \cdot \bar{\eta}^{2}(x, y)\right. \\
& +2 \partial_{34} F\left[u^{*}\right](x, y) \cdot \bar{\eta}(x, y) \cdot{ }^{C} D_{a, x}^{\alpha} \bar{\eta}(x, y) \\
& +2 \partial_{35} F\left[u^{*}\right](x, y) \cdot \bar{\eta}(x, y) \cdot{ }^{C} D_{c, y}^{\alpha} \bar{\eta}(x, y) \\
& +\partial_{44} F\left[u^{*}\right](x, y) \cdot\left({ }^{C} D_{a, x}^{\alpha} \bar{\eta}(x, y)\right)^{2} \\
& +2 \partial_{45} F\left[u^{*}\right](x, y) \cdot{ }^{C} D_{a, x}^{\alpha} \bar{\eta}(x, y) \cdot{ }^{C} D_{c, y}^{\alpha} \bar{\eta}(x, y) \\
& \left.+\partial_{55} F\left[u^{*}\right](x, y) \cdot\left({ }^{C} D_{c, y}^{\alpha} \bar{\eta}(x, y)\right)^{2}\right) d x d y \\
& =\iint_{\bar{D}}\left(\partial_{33} F\left[u^{*}\right](x, y) \cdot h^{2}(x, y)\right. \\
& +2 \partial_{34} F\left[u^{*}\right](x, y) \cdot h(x, y) \cdot{ }^{C} D_{a, x}^{\alpha} h(x, y) \\
& +2 \partial_{35} F\left[u^{*}\right](x, y) \cdot h(x, y) \cdot{ }^{C} D_{c, y}^{\alpha} h(x, y) \\
& +\partial_{44} F\left[u^{*}\right](x, y) \cdot\left({ }^{C} D_{a, x}^{\alpha} h(x, y)\right)^{2} \\
& +2 \partial_{45} F\left[u^{*}\right](x, y) \cdot{ }^{C} D_{a, x}^{\alpha} h(x, y) \cdot{ }^{C} D_{c, y}^{\alpha} h(x, y) \\
& \left.+\partial_{55} F\left[u^{*}\right](x, y) \cdot\left({ }^{C} D_{c, y}^{\alpha} h(x, y)\right)^{2}\right) d x d y \\
& \leq \iint_{\bar{D}}\left((14)^{4} C_{1}(\bar{b}-\bar{a})^{2+2 \alpha}(\bar{d}-\bar{c})^{2+2 \alpha}\right. \\
& +2(14)^{2} C_{2} \bar{C}(\bar{b}-\bar{a})^{2+\alpha}(\bar{d}-\bar{c})^{2+2 \alpha} \\
& +2(14)^{2} C_{3} \bar{C}(\bar{b}-\bar{a})^{2+2 \alpha}(\bar{d}-\bar{c})^{2+\alpha} \\
& +C_{4}(\bar{C})^{2}(\bar{b}-\bar{a})^{2}(\bar{d}-\bar{c})^{2+2 \alpha} \\
& +2 C_{5}(\bar{C})^{2}(\bar{b}-\bar{a})^{2+\alpha}(\bar{d}-\bar{c})^{2+\alpha} \\
& \left.+C_{6}(\bar{C})^{2}(\bar{b}-\bar{a})^{2+2 \alpha}(\bar{d}-\bar{c})^{2}\right) d x d y \\
& \leq(\bar{d}-\bar{c})^{2} \iint_{\bar{D}}\left((14)^{4} C_{1}(\bar{b}-\bar{a})^{2+2 \alpha}(\bar{d}-\bar{c})^{2 \alpha}\right. \\
& +2(14)^{2} C_{2} \bar{C}(\bar{b}-\bar{a})^{2+\alpha}(\bar{d}-\bar{c})^{2 \alpha} \\
& +2(14)^{2} C_{3} \bar{C}(\bar{b}-\bar{a})^{2+2 \alpha}(\bar{d}-\bar{c})^{\alpha}
\end{aligned}
$$

$$
\begin{aligned}
& +C_{4}(\bar{C})^{2}(\bar{b}-\bar{a})^{2}(\bar{d}-\bar{c})^{2 \alpha} \\
& +2 C_{5}(\bar{C})^{2}(\bar{b}-\bar{a})^{2+\alpha}(\bar{d}-\bar{c})^{\alpha} \\
& \left.+C_{6}(\bar{C})^{2}(\bar{b}-\bar{a})^{2+2 \alpha}\right) d x d y<0
\end{aligned}
$$

If $|\bar{d}-\bar{c}| \ll|\bar{b}-\bar{a}| \ll 1$, there arises a contradiction.

Hence, (27) holds. In the same manner, we can get (26).

3.3. The Fractional Variational Problem with Holonomic Constraint. Consider the functional $J$ defined by

$$
\begin{gathered}
J\left(u_{1}(x, y), u_{2}(x, y)\right)=\iint_{D} F\left(x, y, u_{1}(x, y),\right. \\
u_{2}(x, y),{ }^{C} D_{a, x}^{\alpha} u_{1}(x, y),{ }^{C} D_{a, x}^{\alpha} u_{2}(x, y), \\
\left.{ }^{C} D_{c, y}^{\alpha} u_{1}(x, y),{ }^{C} D_{c, y}^{\alpha} u_{2}(x, y)\right) d x d y
\end{gathered}
$$

on the space

$$
\widetilde{U}=\left\{\left(u_{1}(x, y), u_{2}(x, y)\right) \in C^{1}(D) \times C^{1}(D)\right\}
$$

subject to

$$
\begin{aligned}
u_{1}(x, c) & =\varphi_{1}(x), \quad x \in[a, b] ; \\
u_{1}(a, y) & =\psi_{1}(y), \quad y \in[c, d] ; \\
\varphi_{1}(a) & =\psi_{1}(c) ; \\
u_{2}(x, c) & =\varphi_{2}(x), \quad x \in[a, b] ; \\
u_{2}(a, y) & =\psi_{2}(y), \quad y \in[c, d] ; \\
\varphi_{2}(a) & =\psi_{2}(c),
\end{aligned}
$$

where $\varphi_{i} \in A C([a, b]),(i=1,2)$ and $\psi_{i} \in A C([c, d]), \quad(i=$ $1,2)$. For simplicity, we denote

$$
\begin{aligned}
& u(x, y)=\left(u_{1}(x, y), u_{2}(x, y)\right) \\
& { }^{C} D_{a, x}^{\alpha} u(x, y)=\left({ }^{C} D_{a, x}^{\alpha} u_{1}(x, y),{ }^{C} D_{a, x}^{\alpha} u_{2}(x, y)\right) \\
& { }^{C} D_{x, b}^{\alpha} u(x, y)=\left({ }^{C} D_{x, b}^{\alpha} u_{1}(x, y),{ }^{C} D_{x, b}^{\alpha} u_{2}(x, y)\right) \\
& { }^{C} D_{c, y}^{\alpha} u(x, y)=\left({ }^{C} D_{c, y}^{\alpha} u_{1}(x, y),{ }^{C} D_{c, y}^{\alpha} u_{2}(x, y)\right) \\
& { }^{C} D_{y, d}^{\alpha} u(x, y)=\left({ }^{C} D_{y, d}^{\alpha} u_{1}(x, y),{ }^{C} D_{y, d}^{\alpha} u_{2}(x, y)\right) \\
& {[u](x, y)=\left(x, y, u_{1}(x, y), u_{2}(x, y),{ }^{C} D_{a, x}^{\alpha} u_{1}(x, y),\right.} \\
& \left.{ }^{C} D_{a, x}^{\alpha} u_{2}(x, y),{ }^{C} D_{c, y}^{\alpha} u_{1}(x, y),{ }^{C} D_{c, y}^{\alpha} u_{2}(x, y)\right)
\end{aligned}
$$

Assume the Lagrangian in (38) satisfies the following conditions:

(iii) $F: D \times R^{6} \longrightarrow R$ is continuously differentiable with respect to its $i$ th argument, for $i=3,4, \cdots 8$; 
(iv) Given any functions $u_{1}(x, y), u_{2}(x, y) \in D$, the maps

$$
\begin{aligned}
& (x, y) \longmapsto{ }^{C} D_{x, b}^{\alpha} \partial_{4} F\left(x, y, u(x, y),{ }^{C} D_{a, x}^{\alpha} u(x, y),\right. \\
& \left.{ }^{C} D_{c, y}^{\alpha} u(x, y)\right) \\
& (x, y) \longmapsto{ }^{C} D_{y, d}^{\alpha} \partial_{5} F\left(x, y, u(x, y),{ }^{C} D_{a, x}^{\alpha} u(x, y),\right. \\
& \left.{ }^{C} D_{c, y}^{\alpha} u(x, y)\right)
\end{aligned}
$$

are continuous.

We consider the variational problem when in presence of a holonomic constraint. Assume that the admissible functions lie on the surface

$$
g\left(x, y, u_{1}(x, y), u_{2}(x, y)\right)=0
$$

where $g: D \times R^{2} \longrightarrow R$ is continuously differentiable with respect to its $i$ th argument, for $i=3,4$.

Theorem 15. Let $u^{*} \in U$ be a minimizer of $J$ as in (38), under the constraint (43). If

$$
\partial_{4} g\left(x, y, u_{1}^{*}(x, y), u_{2}^{*}(x, y)\right) \neq 0, \quad \forall(x, y) \in D,
$$

then there is a continuous function $\lambda: D \longrightarrow R$ such that

$$
\begin{aligned}
& \partial_{3} F\left[u^{*}\right](x, y)-D_{x, b}^{\alpha} \partial_{5} F\left[u^{*}\right](x, y) \\
&-D_{y, d}^{\alpha} \partial_{7} F\left[u^{*}\right](x, y) \\
&+\lambda(x, y) \partial_{3} g\left(x, y, u_{1}^{*}(x, y), u_{2}^{*}(x, y)\right)=0
\end{aligned}
$$

and

$$
\begin{aligned}
\partial_{4} F\left[u^{*}\right](x, y)-D_{x, b}^{\alpha} \partial_{6} F\left[u^{*}\right](x, y) \\
\quad-D_{y, d}^{\alpha} \partial_{8} F\left[u^{*}\right](x, y) \\
\quad+\lambda(x, y) \partial_{4} g\left(x, y, u_{1}^{*}(x, y), u_{2}^{*}(x, y)\right)=0
\end{aligned}
$$

Proof. Consider a variation of $u^{*}(x, y)$ of type $u^{*}+\varepsilon \eta$, with $|\varepsilon| \ll 1$, and $\eta=\left(\eta_{1}, \eta_{2}\right) \in C^{1}(D) \times C^{1}(D)$, satisfying the boundary conditions $\left.\eta_{i}(x, y)\right|_{\partial D}=0,(i=1,2)$. By

$$
\partial_{4} g\left(x, y, u_{1}^{*}(x, y), u_{2}^{*}(x, y)\right) \neq 0, \quad \forall(x, y) \in D
$$

and the implicit function theorem, there exists a subfamily of variations satisfying restriction equation (43). That is, there exists a unique function $\eta_{2}\left(\varepsilon, \eta_{1}\right)$ such that $\left(u_{1}^{*}+\varepsilon \eta_{1}, u_{2}^{*}+\varepsilon \eta_{2}\right)$ satisfies (43). Hence, for all $(x, y) \in D$, we have

$$
\begin{aligned}
& g\left(x, y, u_{1}^{*}(x, y)+\varepsilon \eta_{1}(x, y), u_{2}^{*}(x, y)+\varepsilon \eta_{2}(x, y)\right) \\
& \quad=0 .
\end{aligned}
$$
we get

Differentiating (48) with respect to $\varepsilon$ and putting $\varepsilon=0$,

$$
\begin{aligned}
& \partial_{3} g\left(x, y, u_{1}^{*}(x, y), u_{2}^{*}(x, y)\right) \eta_{1}(x, y) \\
& \quad+\partial_{4} g\left(x, y, u_{1}^{*}(x, y), u_{2}^{*}(x, y)\right) \eta_{2}(x, y)=0 .
\end{aligned}
$$

Define the function

$$
\begin{aligned}
& \lambda(x, y) \\
& =-\frac{\partial_{4} F\left[u^{*}(x, y)\right]-D_{x, b}^{\alpha} \partial_{6} F\left[u^{*}(x, y)\right]-D_{y, d}^{\alpha} \partial_{8} F\left[u^{*}(x, y)\right]}{\partial_{4} g\left(x, y, u_{1}^{*}(x, y), u_{2}^{*}(x, y)\right)}
\end{aligned}
$$

On the other hand, since $u^{*}$ is a minimizer of $J$, the first variation of $J$ must vanish;

$$
\begin{aligned}
& \iint_{D}\left(\partial_{3} F\left[u^{*}(x, y)\right] \cdot \eta_{1}(x, y)+\partial_{4} F\left[u^{*}(x, y)\right]\right. \\
& \cdot \eta_{2}(x, y)+\partial_{5} F\left[u^{*}(x, y)\right] \cdot{ }^{C} D_{a, x}^{\alpha} \eta_{1}(x, y) \\
& +\partial_{6} F\left[u^{*}(x, y)\right] \cdot{ }^{C} D_{a, x}^{\alpha} \eta_{2}(x, y)+\partial_{7} F\left[u^{*}(x, y)\right] \\
& .{ }^{C} D_{c, y}^{\alpha} \eta_{1}(x, y)+\partial_{8} F\left[u^{*}(x, y)\right] \\
& \left..{ }^{C} D_{c, y}^{\alpha} \eta_{2}(x, y)\right) d x d y=0
\end{aligned}
$$
obtain

Integrating by parts and $\left.\eta_{i}(x, y)\right|_{\partial D}=0,(i=1,2)$, we

$$
\begin{aligned}
& \iint_{D}\left\{\left(\partial_{3} F\left[u^{*}(x, y)\right]-D_{x, b}^{\alpha} \partial_{5} F\left[u^{*}(x, y)\right]\right.\right. \\
& \left.-D_{y, d}^{\alpha} \partial_{7} F\left[u^{*}(x, y)\right]\right) \eta_{1}(x, y)+\left(\partial_{4} F\left[u^{*}(x, y)\right]\right. \\
& \left.-D_{x, b}^{\alpha} \partial_{6} F\left[u^{*}(x, y)\right]-D_{y, d}^{\alpha} \partial_{8} F\left[u^{*}(x, y)\right]\right) \\
& \left.\cdot \eta_{2}(x, y)\right\} d x d y+\iint_{D}\left\{\frac { \partial } { \partial x } \left(\eta_{1}(x, y)\right.\right. \\
& \left.\cdot I_{x, b}^{1-\alpha} \partial_{5} F\left[u^{*}(x, y)\right]\right)+\frac{\partial}{\partial y}\left(\eta_{1}(x, y)\right. \\
& \left.\left.\cdot I_{y, d}^{1-\alpha} \partial_{7} F\left[u^{*}(x, y)\right]\right)\right\} d x d y \\
& +\iint_{D}\left\{\frac{\partial}{\partial x}\left(\eta_{2}(x, y) \cdot I_{x, b}^{1-\alpha} \partial_{6} F\left[u^{*}(x, y)\right]\right)\right. \\
& +\frac{\partial}{\partial y}\left(\eta_{2}(x, y)\right. \\
& \left.\left.\cdot I_{y, d}^{1-\alpha} \partial_{8} F\left[u^{*}(x, y)\right]\right)\right\} d x d y \\
& =\iint_{D}\left\{\left(\partial_{3} F\left[u^{*}(x, y)\right]-D_{x, b}^{\alpha} \partial_{5} F\left[u^{*}(x, y)\right]\right.\right. \\
& \left.-D_{y, d}^{\alpha} \partial_{7} F\left[u^{*}(x, y)\right]\right) \eta_{1}(x, y)+\left(\partial_{4} F\left[u^{*}(x, y)\right]\right. \\
& \left.-D_{x, b}^{\alpha} \partial_{6} F\left[u^{*}(x, y)\right]-D_{y, d}^{\alpha} \partial_{8} F\left[u^{*}(x, y)\right]\right)
\end{aligned}
$$




$$
\begin{aligned}
& \left.\cdot \eta_{2}(x, y)\right\} d x d y+\oint_{\partial D}\left(-\eta_{1}(x, y)\right. \\
& \left.\cdot I_{y, d}^{1-\alpha} \partial_{7} F\left[u^{*}\right](x, y)\right) d x+\left(\eta_{1}(x, y)\right. \\
& \left.\cdot I_{x, b}^{1-\alpha} \partial_{5} F\left[u^{*}\right](x, y)\right) d y+\oint_{\partial D}\left(-\eta_{2}(x, y)\right. \\
& \left.\cdot I_{y, d}^{1-\alpha} \partial_{8} F\left[u^{*}\right](x, y)\right) d x+\left(\eta_{2}(x, y)\right. \\
& \left.\cdot I_{x, b}^{1-\alpha} \partial_{6} F\left[u^{*}\right](x, y)\right) d y \\
& =\iint_{D}\left\{\left(\partial_{3} F\left[u^{*}(x, y)\right]-D_{x, b}^{\alpha} \partial_{5} F\left[u^{*}(x, y)\right]\right.\right. \\
& \left.-D_{y, d}^{\alpha} \partial_{7} F\left[u^{*}(x, y)\right]\right) \eta_{1}(x, y)+\left(\partial_{4} F\left[u^{*}(x, y)\right]\right. \\
& \left.-D_{x, b}^{\alpha} \partial_{6} F\left[u^{*}(x, y)\right]-D_{y, d}^{\alpha} \partial_{8} F\left[u^{*}(x, y)\right]\right) \\
& \left.\cdot \eta_{2}(x, y)\right\} d x d y=\iint_{D}\left(\partial_{3} F\left[u^{*}\right]\right. \\
& \cdot(x, y)-D_{x, b}^{\alpha} \partial_{5} F\left[u^{*}\right](x, y)-D_{y, d}^{\alpha} \partial_{7} F\left[u^{*}\right] \\
& \left.\cdot(x, y)+\lambda(x, y) \partial_{3} g\left(x, y, u^{*}(x, y)\right)\right) \eta_{1}(x, \\
& y) d y=0
\end{aligned}
$$

Since $\eta_{1}(x, y)$ is arbitrary, we have that $u^{*}$ is a solution of the equation

$$
\begin{aligned}
\partial_{3} F\left[u^{*}\right](x, y)-D_{x, b}^{\alpha} \partial_{5} F\left[u^{*}\right](x, y) \\
\quad-D_{y, d}^{\alpha} \partial_{7} F\left[u^{*}\right](x, y) \\
\quad+\lambda(x, y) \partial_{3} g\left(x, y, u^{*}(x, y)\right)=0
\end{aligned}
$$

At the same time, by (50), we obtain the second condition

$$
\begin{aligned}
\partial_{4} F\left[u^{*}\right](x, y)-D_{x, b}^{\alpha} \partial_{6} F\left[u^{*}\right](x, y) \\
\quad-D_{y, d}^{\alpha} \partial_{8} F\left[u^{*}\right](x, y) \\
\quad+\lambda(x, y) \partial_{4} g\left(x, y, u^{*}(x, y)\right)=0
\end{aligned}
$$

3.4. Isoperimetric Problem. Let $c$ be constant and $N: D \times$ $R^{3} \longrightarrow R$ be a continuously differentiable function with respect to its $i$-th argument, for $i=3,4,5$. For any function $u \in C^{1}(D)$, the maps

$$
\begin{aligned}
& (x, y) \longmapsto{ }^{C} D_{x, b}^{\alpha} \partial_{4} N\left(x, y, u(x, y),{ }^{C} D_{a, x}^{\alpha} u(x, y),\right. \\
& \left.{ }^{C} D_{c, y}^{\alpha} u(x, y)\right)
\end{aligned}
$$

and

$$
\begin{aligned}
& (x, y) \longmapsto{ }^{C} D_{y, d}^{\alpha} \partial_{5} N\left(x, y, u(x, y),{ }^{C} D_{a, x}^{\alpha} u(x, y),\right. \\
& \left.{ }^{C} D_{c, y}^{\alpha} u(x, y)\right)
\end{aligned}
$$

are continuous.

We now consider a new isoperimetric type problem with the isoperimetric constraint as follows:

$$
\begin{gathered}
n(u(x, y))=\iint_{D} N\left(x, y, u(x, y),{ }^{C} D_{a, x}^{\alpha} u(x, y),\right. \\
\left.{ }^{C} D_{c, y}^{\alpha} u(x, y)\right) d x d y=c
\end{gathered}
$$

Theorem 16. Suppose that $u^{*}$ is a local minimizer for $J$ as in (25) on the space D, subject to the integral constraint (57). If $u^{*}$ is not an extremal of $n(u)$, then there exists a real $\lambda$ such that $u^{*}$ is a solution of the equation

$$
\begin{gathered}
\partial_{3} g\left[u^{*}\right](x, y)-D_{x, b}^{\alpha} \partial_{4} g\left[u^{*}\right](x, y) \\
-D_{y, d}^{\alpha} \partial_{5} g\left[u^{*}\right](x, y)=0
\end{gathered}
$$

where the function $g: D \times R^{3} \longrightarrow R$ is defined by $g=F+\lambda N$.

Proof. Consider a variation of two parameters $u^{*}+\varepsilon_{1} \eta_{1}(x$, $y)+\varepsilon_{2} \eta_{2}(x, y)$, with $\left|\varepsilon_{i}\right| \ll 1$ and $\eta_{i}(x, y) \in C^{1}(D)$ satisfying $\left.\eta_{i}(x, y)\right|_{\partial D}=0, i=1,2$. Define the functions $i$ and $j$ with two parameters $\left(\varepsilon_{1}, \varepsilon_{2}\right)$ in a neighborhood of zero as

$$
i\left(\varepsilon_{1}, \varepsilon_{2}\right)=n\left(u^{*}+\varepsilon_{1} \eta_{1}(x, y)+\varepsilon_{2} \eta_{2}(x, y)\right)-c
$$

and

$$
j\left(\varepsilon_{1}, \varepsilon_{2}\right)=J\left(u^{*}+\varepsilon_{1} \eta_{1}(x, y)+\varepsilon_{2} \eta_{2}(x, y)\right)
$$

When $\varepsilon_{1}=\varepsilon_{2}=0$, the derivative $\partial i / \partial \varepsilon_{2}$ is

$$
\begin{aligned}
& \frac{\partial i}{\partial \varepsilon_{2}}(0,0)=\iint_{D}\left(\partial_{3} N\left[u^{*}(x, y)\right] \cdot \eta_{2}(x, y)\right. \\
& \quad+\partial_{4} N\left[u^{*}(x, y)\right] \cdot{ }^{C} D_{a, x}^{\alpha} \eta_{2}(x, y) \\
& \left.\quad+\partial_{5} N\left[u^{*}(x, y)\right] \cdot{ }^{C} D_{c, y}^{\alpha} \eta_{2}(x, y)\right) d x d y
\end{aligned}
$$



obtain

Integrating by parts and $\left.\eta_{i}(x, y)\right|_{\partial D}=0,(i=1,2)$, we

$$
\begin{aligned}
& \frac{\partial i}{\partial \varepsilon_{2}}(0,0)=\iint_{D}\left(\partial_{3} N\left[u^{*}(x, y)\right]\right. \\
& \left.-D_{x, b}^{\alpha} \partial_{4} N\left[u^{*}(x, y)\right]-D_{y, d}^{\alpha} \partial_{5} N\left[u^{*}(x, y)\right]\right) \\
& \cdot \eta_{2}(x, y) d x d y \\
& +\iint_{D}\left\{\frac{\partial}{\partial x}\left(I_{x, b}^{1-\alpha} \partial_{4} N\left[u^{*}(x, y)\right] \cdot \eta_{2}(x, y)\right)\right. \\
& +\frac{\partial}{\partial y}\left(I_{y, d}^{1-\alpha} \partial_{5} N\left[u^{*}(x, y)\right]\right. \\
& \left.\left.\quad \cdot \eta_{2}(x, y)\right)\right\} d x d y \\
& \quad=\iint_{D}\left(\partial_{3} N\left[u^{*}(x, y)\right]-D_{x, b}^{\alpha} \partial_{4} N\left[u^{*}(x, y)\right]\right. \\
& \left.\quad-D_{y, d}^{\alpha} \partial_{5} N\left[u^{*}(x, y)\right]\right) \eta_{2}(x, y) d x d y \\
& +\oint_{\partial D}\left(-\eta_{2}(x, y) I_{y, d}^{1-\alpha} \partial_{5} N\left[u^{*}\right](x, y)\right) d x \\
& +\eta_{2}(x, y) I_{x, b}^{1-\alpha} \partial_{4} N\left[u^{*}\right](x, y) d y \\
& =
\end{aligned}
$$

Since $u^{*}$ is not an extremal of $n(u)$, there exists a function $\eta_{2}(x, y)$ such that $\left(\partial i / \partial \varepsilon_{2}\right)(0,0) \neq 0$. An application of the implicit function theorem implies that there exists a subfamily of variations satisfying the integral constraint.

On the other hand, $(0,0)$ is a minimize of $j$, under the restriction $i(\cdot, \cdot)=0$, and we just proved that $\nabla i(0,0) \neq 0$. By the Lagrange multiplier rule, there exists a real number $\lambda$ such that $\nabla(j+\lambda i)(0,0)=(0,0)$. In particular, $\left(\partial / \partial \varepsilon_{1}\right)(j+$ $\lambda i)(0,0)=0$. Since $\left.\eta_{1}(x, y)\right|_{\partial D}=0$, we have

$$
\begin{aligned}
& \iint_{D}\left(\partial_{3} g\left[u^{*}\right](x, y)-D_{x, b}^{\alpha} \partial_{4} g\left[u^{*}\right](x, y)\right. \\
& \left.-D_{y, d}^{\alpha} \partial_{5} g\left[u^{*}\right](x, y)\right) \eta_{1}(x, y) d x d y \\
& +\oint_{\partial D}\left(-\eta_{1}(x, y) I_{y, d}^{1-\alpha} \partial_{5} N\left[u^{*}\right](x, y)\right) d x \\
& +\eta_{1}(x, y) I_{x, b}^{1-\alpha} \partial_{4} N\left[u^{*}\right](x, y) d y \\
& \quad=\iint_{D}\left(\partial_{3} g\left[u^{*}\right](x, y)-D_{x, b}^{\alpha} \partial_{4} g\left[u^{*}\right](x, y)\right. \\
& \left.\quad-D_{y, d}^{\alpha} \partial_{5} g\left[u^{*}\right](x, y)\right) \eta_{1}(x, y) d x d y=0
\end{aligned}
$$

Hence,

$$
\begin{gathered}
\partial_{3} g\left[u^{*}\right](x, y)-D_{x, b}^{\alpha} \partial_{4} g\left[u^{*}\right](x, y) \\
-D_{y, d}^{\alpha} \partial_{5} g\left[u^{*}\right](x, y)=0
\end{gathered}
$$

This ends the proof.

Theorem 17. Suppose that the functions $F(x, y, u(x, y)$, $\left.{ }^{C} D_{a, x}^{\alpha} u(x, y),{ }^{C} D_{c, y}^{\alpha} u(x, y)\right)$ and $N\left(x, y, u(x, y),{ }^{C} D_{a, x}^{\alpha} u(x, y)\right.$, $\left.{ }^{C} D_{c, y}^{\alpha} u(x, y)\right)$ as in (25) and (57) are convex in $D \times R^{3}$. Let $\lambda \geq 0$ be a constant and $g=F+\lambda N$. Then, each solution $u^{*}$ of the fractional Euler-Lagrange equation

$$
\begin{gathered}
\partial_{3} g\left[u^{*}\right](x, y)-D_{x, b}^{\alpha} \partial_{4} g\left[u^{*}\right](x, y) \\
-D_{y, d}^{\alpha} \partial_{5} g\left[u^{*}\right](x, y)=0
\end{gathered}
$$

minimizes $J$ in $U$, subject to the integral constraint (57).

Proof. Consider a variation of $u^{*}(x, y)$ of type $u^{*}+\varepsilon \eta$, with $|\varepsilon| \ll 1$ and $\eta \in C^{1}(D)$, satisfying the boundary conditions $\left.\eta(x, y)\right|_{\partial D}=0$. Noting that $g$ is convex,

$$
\begin{aligned}
& \iint_{D} g\left(x, y, u^{*}(x, y)+\varepsilon \eta(x, y),{ }^{C} D_{a, x}^{\alpha} u^{*}(x, y)\right. \\
& +\varepsilon^{C} D_{a, x}^{\alpha} \eta(x, y),{ }^{C} D_{c, y}^{\alpha} u^{*}(x, y) \\
& \left.+\varepsilon^{C} D_{c, y}^{\alpha} \eta(x, y)\right) d x d y-\iint_{D} g(x, y, \\
& u^{*}(x, y),{ }^{C} D_{a, x}^{\alpha} u^{*}(x, y) \text {, } \\
& \left.{ }^{C} D_{c, y}^{\alpha} u^{*}(x, y)\right) d x d y \geq \iint_{D} \partial_{3} g\left[u^{*}\right] \\
& \cdot(x, y) \varepsilon \eta(x, y)+\partial_{4} g\left[u^{*}\right](x, y) \varepsilon^{C} D_{a, x}^{\alpha} \eta(x, y) \\
& +\partial_{5} g\left[u^{*}\right](x, y) \varepsilon^{C} D_{c, y}^{\alpha}(\eta(x, y)) d x d y \\
& =\iint_{D}\left(\partial_{3} g\left[u^{*}\right](x, y)-D_{x, b}^{\alpha} \partial_{4} g\left[u^{*}\right](x, y)\right. \\
& \left.-D_{y, d}^{\alpha} \partial_{5} g\left[u^{*}\right](x, y)\right) \varepsilon \eta(x, y) d x d y \\
& +\iint_{D}\left(\frac{\partial}{\partial x}\left(I_{x, b}^{1-\alpha} \partial_{4} g\left[u^{*}\right](x, y) \varepsilon \eta(x, y)\right)\right. \\
& \left.+\frac{\partial}{\partial y}\left(I_{y, d}^{1-\alpha} \partial_{5} g\left[u^{*}\right](x, y) \varepsilon \eta(x, y)\right)\right) d x d y \\
& =\iint_{D}\left(\partial_{3} g\left[u^{*}\right](x, y)-D_{x, b}^{\alpha} \partial_{4} g\left[u^{*}\right](x, y)\right. \\
& \left.-D_{y, d}^{\alpha} \partial_{5} g\left[u^{*}\right](x, y)\right) \varepsilon \eta(x, y) d x d y \\
& +\varepsilon \oint_{\partial D}\left(-I_{y, d}^{1-\alpha} \partial_{5} g\left[u^{*}\right](x, y) \eta(x, y)\right) d x \\
& +I_{x, b}^{1-\alpha} \partial_{4} g\left[u^{*}\right](x, y) \eta(x, y) d y=0
\end{aligned}
$$


We have

$$
\begin{aligned}
& \iint_{D} F\left(x, y, u^{*}(x, y)+\varepsilon \eta(x, y),{ }^{C} D_{a, x}^{\alpha} u^{*}(x, y)\right. \\
& +\varepsilon^{C} D_{a, x}^{\alpha} \eta(x, y),{ }^{C} D_{c, y}^{\alpha} u^{*}(x, y) \\
& \left.+\varepsilon^{C} D_{c, y}^{\alpha} \eta(x, y)\right) d x d y+\iint_{D} \lambda N(x, \\
& y, u^{*}(x, y)+\varepsilon \eta(x, y),{ }^{C} D_{a, x}^{\alpha} u^{*}(x, y) \\
& +\varepsilon^{C} D_{a, x}^{\alpha} \eta(x, y),{ }^{C} D_{c, y}^{\alpha} u^{*}(x, y) \\
& \left.+\varepsilon^{C} D_{c, y}^{\alpha} \eta(x, y)\right) d x d y \geq \iint_{D} F(x, y, \\
& u^{*}(x, y),{ }^{C} D_{a, x}^{\alpha} u^{*}(x, y), \\
& \left.{ }^{C} D_{c, y}^{\alpha} u^{*}(x, y)\right) d x d y+\iint_{D} \lambda N(x, y, \\
& u^{*}(x, y),{ }^{C} D_{a, x}^{\alpha} u^{*}(x, y), \\
& \left.{ }^{C} D_{c, y}^{\alpha} u^{*}(x, y)\right) d x d y
\end{aligned}
$$

According to the integral constraint, we obtain

$$
\begin{aligned}
& \iint_{D} F\left(x, y, u^{*}(x, y)+\varepsilon \eta(x, y),{ }^{C} D_{a, x}^{\alpha} u^{*}(x, y)\right. \\
& \quad+\varepsilon^{C} D_{a, x}^{\alpha} \eta(x, y),{ }^{C} D_{c, y}^{\alpha} u^{*}(x, y) \\
& \left.\quad+\varepsilon^{C} D_{c, y}^{\alpha} \eta(x, y)\right) d x d y+\lambda c \\
& \quad \geq \iint_{D} F\left(x, y, u^{*}(x, y),{ }^{C} D_{a, x}^{\alpha} u^{*}(x, y),\right. \\
& \left.\quad{ }^{C} D_{c, y}^{\alpha} u^{*}(x, y)\right) d x d y+\lambda c
\end{aligned}
$$

As a consequence,

$$
\begin{aligned}
& \iint_{D} F\left(x, y, u^{*}(x, y)+\varepsilon \eta(x, y),{ }^{C} D_{a, x}^{\alpha} u^{*}(x, y)\right. \\
& \quad+\varepsilon^{C} D_{a, x}^{\alpha} \eta(x, y),{ }^{C} D_{c, y}^{\alpha} u^{*}(x, y) \\
& \left.\quad+\varepsilon^{C} D_{c, y}^{\alpha} \eta(x, y)\right) d x d y \geq \iint_{D} F(x, y, \\
& u^{*}(x, y),{ }^{C} D_{a, x}^{\alpha} u^{*}(x, y), \\
& \left.\quad{ }^{C} D_{c, y}^{\alpha} u^{*}(x, y)\right) d x d y
\end{aligned}
$$

This ends the proof.

3.5. Noether Symmetry. Suppose that the configuration of a dynamical system is

$$
F\left(x, y, u(x, y),{ }^{C} D_{a, x}^{\alpha} u(x, y),{ }^{C} D_{c, y}^{\alpha} u(x, y)\right)
$$

The action is defined by (25); i.e.,

$$
\begin{gathered}
J=\iint_{D} F\left(x, y, u(x, y),{ }^{C} D_{a, x}^{\alpha} u(x, y),\right. \\
\left.{ }^{C} D_{c, y}^{\alpha} u(x, y)\right) d x d y
\end{gathered}
$$

The isochronal variation principle

$$
\delta J=0
$$

with fixed end-point conditions

$$
\left.\delta u\right|_{\partial D}=0
$$

is called the Hamilton principle on the action. From the principle equations (72)-(73) and Theorem 9, it is easy to obtain Euler-Lagrange equations $\left(20^{\prime}\right)$. Let us introduce the infinitesimal transformations of the one-parameter finite transformation group $G$; their expansion formulas are

$$
\begin{aligned}
\bar{x} & =x+\varepsilon \xi^{x}(x, y, u), \\
\bar{y} & =y+\varepsilon \xi^{y}(x, y, u), \\
\bar{u}(\bar{x}, \bar{y}) & =u(x, y)+\varepsilon \xi^{u}(x, y, u)
\end{aligned}
$$

where $\varepsilon$ is infinitesimal parameter; $\xi^{x}, \xi^{y}$, and $\xi^{u}$ are infinitesimal generators or generating functions of the infinitesimal transformations. We introduce the following notation [14], $\Delta x=\left.(d / d \varepsilon)\right|_{\varepsilon=0}(\bar{x}-x), \Delta y=\left.(d / d \varepsilon)\right|_{\varepsilon=0}(\bar{y}-y)$, and $\Delta u=$ $\left.(d / d \varepsilon)\right|_{\varepsilon=0}(\bar{u}(\bar{x}, \bar{y})-u(x, y))$.

So we have

$$
\begin{aligned}
\Delta u= & \left.\frac{d}{d \varepsilon}\right|_{\varepsilon=0}(\bar{u}(\bar{x}, \bar{y})-u(x, y)) \\
= & \left.\frac{d}{d \varepsilon}\right|_{\varepsilon=0}(\bar{u}(\bar{x}, \bar{y})-\bar{u}(x, \bar{y})) \\
& +\left.\frac{d}{d \varepsilon}\right|_{\varepsilon=0}(\bar{u}(x, \bar{y})-\bar{u}(x, y)) \\
& +\left.\frac{d}{d \varepsilon}\right|_{\varepsilon=0}(\bar{u}(x, y)-u(x, y)) \\
= & u_{x} \Delta x+u_{y} \Delta y+\delta u
\end{aligned}
$$

and

$$
\delta u=\Delta u-u_{x} \Delta x-u_{y} \Delta y=\xi^{u}-u_{x} \xi^{x}-u_{y} \xi^{y}
$$

Theorem 18. Let $u \in A C(D)$ and $G$ be a local one-parameter group of transformations by (74). Then

$$
\begin{aligned}
\Delta^{C} D_{a, x}^{\alpha} u(x, y)= & { }^{C} D_{a, x}^{\alpha} \delta u+D_{a, x}^{\alpha} u_{x}(x, y) \\
& \cdot \xi^{x}(x, y, u)+\frac{d}{d y}{ }^{C} D_{a, x}^{\alpha} u(x, y) \\
& \cdot \xi^{y}(x, y, u)+\frac{1}{\Gamma(1-\alpha)} \frac{u_{x}(a, y)}{(x-a)^{\alpha}} \\
& \cdot \xi^{x}(x, y, u)
\end{aligned}
$$


and

$$
\begin{aligned}
\Delta^{C} D_{c, y}^{\alpha} u(x, y)= & { }^{C} D_{c, y}^{\alpha} \delta u+D_{c, y}^{\alpha} u_{y}(x, y) \\
& \cdot \xi^{y}(x, y, u)+\frac{d}{d x}{ }^{C} D_{c, y}^{\alpha} u(x, y) \\
& \cdot \xi^{x}(x, y, u)+\frac{1}{\Gamma(1-\alpha)} \frac{u_{y}(x, c)}{(y-c)^{\alpha}} \\
& \cdot \xi^{y}(x, y, u)
\end{aligned}
$$

Proof. By the definitions, we have

$$
\begin{aligned}
& \Delta^{C} D_{a, x}^{\alpha} u(x, y)=\left.\frac{d}{d \varepsilon_{\sigma}}\right|_{\varepsilon_{\sigma}=0}\left[{ }^{C} D_{\bar{a}, \bar{x}}^{\alpha} \bar{u}(\bar{x}, \bar{y})\right. \\
& \left.-{ }^{C} D_{a, x}^{\alpha} u(x, y)\right]=\left.\frac{1}{\Gamma(1-\alpha)} \frac{d}{d \varepsilon}\right|_{\varepsilon=0} \\
& \cdot\left[\int_{\bar{a}}^{\bar{x}}(\bar{x}-s)^{-\alpha} \bar{u}_{s}(s, \bar{y}) d s\right. \\
& \left.-\int_{a}^{x}(x-s)^{-\alpha} u_{s}(s, y) d s\right]=\left.\frac{1}{\Gamma(1-\alpha)} \frac{d}{d \varepsilon}\right|_{\varepsilon=0} \\
& \cdot\left[\int_{\bar{a}}^{a}(\bar{x}-s)^{-\alpha} \bar{u}_{s}(s, \bar{y}) d s\right. \\
& +\int_{a}^{\bar{x}}(\bar{x}-s)^{-\alpha} \bar{u}_{s}(s, \bar{y}) d s \\
& -\int_{a}^{x}(x-s)^{-\alpha} \bar{u}_{s}(s, \bar{y}) d s \\
& +\int_{a}^{x}(x-s)^{-\alpha} \bar{u}_{s}(s, \bar{y}) d s \\
& -\int_{a}^{x}(x-s)^{-\alpha} \bar{u}_{s}(s, y) d s \\
& +\int_{a}^{x}(x-s)^{-\alpha} \bar{u}_{s}(s, y) d s \\
& \left.-\int_{a}^{x}(x-s)^{-\alpha} u_{s}(s, y) d s\right]={ }^{C} D_{a, x}^{\alpha} \delta u \\
& +\left.\frac{1}{\Gamma(1-\alpha)} \frac{d}{d \varepsilon}\right|_{\varepsilon=0} \int_{\bar{a}}^{a}(\bar{x}-s)^{-\alpha} \bar{u}_{s}(s, \bar{y}) d s \\
& +\left.\frac{1}{\Gamma(1-\alpha)} \frac{d}{d \varepsilon}\right|_{\varepsilon=0}\left[\int_{a}^{\bar{x}}(\bar{x}-s)^{-\alpha} \bar{u}_{s}(s, \bar{y}) d s\right. \\
& \left.-\int_{a}^{x}(x-s)^{-\alpha} \bar{u}_{s}(s, \bar{y}) d s\right]+\left.\frac{1}{\Gamma(1-\alpha)} \frac{d}{d \varepsilon}\right|_{\varepsilon=0} \\
& \cdot\left[\int_{a}^{x}(x-s)^{-\alpha} \bar{u}_{s}(s, \bar{y}) d s\right. \\
& \left.-\int_{a}^{x}(x-s)^{-\alpha} \bar{u}_{s}(s, y) d s\right]={ }^{C} D_{a, x}^{\alpha} \delta u
\end{aligned}
$$$$
+\left.\frac{1}{\Gamma(1-\alpha)} \frac{d}{d \varepsilon}\right|_{\varepsilon=0} \int_{\bar{a}}^{a}(\bar{x}-s)^{-\alpha} \bar{u}_{s}(s, \bar{y}) d s
$$$$
+\left.\frac{d}{d \bar{x}} \frac{d \bar{x}}{d \varepsilon}\right|_{\varepsilon=0}\left[I_{a, \bar{x}}^{\alpha} \bar{u}_{x}(\bar{x}, \bar{y})-I_{a, x}^{\alpha} \bar{u}_{x}(x, \bar{y})\right]
$$$$
+\left.\frac{d}{d \bar{y}} \frac{d \bar{y}}{d \varepsilon}\right|_{\varepsilon=0}\left[{ }^{C} D_{a, x}^{\alpha} \bar{u}(x, \bar{y})-{ }^{C} D_{a, x}^{\alpha} \bar{u}(x, y)\right]
$$$$
={ }^{C} D_{a, x}^{\alpha} \delta u+D_{a, x}^{\alpha} u_{x}(x, y) \cdot \xi^{x}(x, y, u)+\frac{d}{d y}
$$$$
\cdot{ }^{C} D_{a, x}^{\alpha} u(x, y) \cdot \xi^{y}(x, y, u)+\frac{1}{\Gamma(1-\alpha)} \frac{u_{x}(a, y)}{(x-a)^{\alpha}}
$$$$
\cdot \xi^{x}(x, y, u)
$$

Analogously, we obtain

$$
\begin{aligned}
\Delta^{C} D_{c, y}^{\alpha} u(x, y)= & { }^{C} D_{c, y}^{\alpha} \delta u+D_{c, y}^{\alpha} u_{y}(x, y) \\
& \cdot \xi^{y}(x, y, u)+\frac{d}{d x}{ }^{C} D_{c, y}^{\alpha} u(x, y) \\
& \cdot \xi^{x}(x, y, u)+\frac{1}{\Gamma(1-\alpha)} \frac{u_{y}(x, c)}{(y-c)^{\alpha}} \\
& \cdot \xi^{y}(x, y, u)
\end{aligned}
$$

Under the infinitesimal transformations, the action equation (25) will be transformed into

$$
\begin{gathered}
J(\bar{S})=\iint_{\bar{D}} F\left(\bar{x}, \bar{y}, \bar{u}(\bar{x}, \bar{y}),{ }^{C} D_{\bar{a}, \bar{x}}^{\alpha} \bar{u}(\bar{x}, \bar{y}),\right. \\
\left.{ }^{C} D_{\bar{c}, \bar{y}}^{\alpha} \bar{u}(\bar{x}, \bar{y})\right) d \bar{x} d \bar{y}
\end{gathered}
$$

The difference of the fractional action before and after the infinitesimal transformation is

$$
\begin{gathered}
J(\bar{S})-J(S)=\iint_{\bar{D}} F\left(\bar{x}, \bar{y}, \bar{u}(\bar{x}, \bar{y}),{ }^{C} D_{\bar{a}, \bar{x}}^{\alpha} \bar{u}(\bar{x}, \bar{y}),\right. \\
\left.{ }^{C} D_{\bar{c}, \bar{y}}^{\alpha} \bar{u}(\bar{x}, \bar{y})\right) d \bar{x} d \bar{y}-\iint_{D} F(x, y, \\
\left.u(x, y),{ }^{C} D_{a, x}^{\alpha} u(x, y),{ }^{C} D_{c, y}^{\alpha} u(x, y)\right) d x d y
\end{gathered}
$$

where the space $\bar{S}$ is adjacent to the space $S$.

Moreover,

$$
\begin{aligned}
& \Delta J=\left.\frac{d}{d \varepsilon}\right|_{\varepsilon=0}\left[\int \int _ { \overline { D } } F \left(\bar{x}, \bar{y}, \bar{u}(\bar{x}, \bar{y}),{ }^{C} D_{\bar{a}, \bar{x}}^{\alpha} \bar{u}(\bar{x}, \bar{y}),\right.\right. \\
& \left.{ }^{C} D_{\bar{c}, \bar{y}}^{\alpha} \bar{u}(\bar{x}, \bar{y})\right) d \bar{x} d \bar{y}-\iint_{D} F(x, y, \\
& \left.\left.u(x, y),{ }^{C} D_{a, x}^{\alpha} u(x, y),{ }^{C} D_{c, y}^{\alpha} u(x, y)\right) d x d y\right] \\
& =\left.\frac{d}{d \varepsilon}\right|_{\varepsilon=0} \iint_{D}\left[F \left(\bar{x}, \bar{y}, \bar{u}(\bar{x}, \bar{y}),{ }^{C} D_{\bar{a}, \bar{x}}^{\alpha} \bar{u}(\bar{x}, \bar{y}),\right.\right.
\end{aligned}
$$




$$
\begin{aligned}
& \left.{ }^{C} D_{\bar{c}, \bar{y}}^{\alpha} \bar{u}(\bar{x}, \bar{y})\right) \operatorname{det}\left(\frac{\partial(\bar{x}, \bar{y})}{\partial(x, y)}\right) \\
& -F\left(x, y, u(x, y),{ }^{C} D_{a, x}^{\alpha} u(x, y)\right. \\
& \left.\left.{ }^{C} D_{c, y}^{\alpha} u(x, y)\right)\right] d x d y=\iint_{D}\left[\partial_{1} F \Delta x\right. \\
& +\partial_{2} F \Delta y+\partial_{3} F \Delta u+\partial_{4} F \Delta^{C} D_{a, x}^{\alpha} u(x, y) \\
& \left.+\partial_{5} F \Delta^{C} D_{c, y}^{\alpha} u(x, y)+F \cdot \operatorname{div}(\bar{X})\right] d x d y
\end{aligned}
$$

where

$$
\operatorname{div}(\bar{X})=\left.\frac{d}{d \varepsilon_{\sigma}} \operatorname{det}\left(\frac{\partial(\bar{x}, \bar{y})}{\partial(x, y)}\right)\right|_{\varepsilon_{\sigma}=0}
$$

By Theorem 9 and relation (76), we get

$$
\begin{aligned}
\Delta J & =\iint_{D}\left[\partial_{1} F \xi^{x}+\partial_{2} F \xi^{y}+\partial_{3} F \xi^{u}+\partial_{4} F \cdot\left({ }^{C} D_{a, x}^{\alpha} \delta u\right.\right. \\
& +D_{a, x}^{\alpha} u_{x} \cdot \xi^{x}+\frac{d}{d y}{ }^{C} D_{a, x}^{\alpha} u \cdot \xi^{y} \\
& \left.+\frac{1}{\Gamma(1-\alpha)} \frac{u_{x}(a, y)}{(x-a)^{\alpha}} \cdot \xi^{x}\right)+\partial_{5} F \cdot\left({ }^{C} D_{c, y}^{\alpha} \delta u\right. \\
& +D_{c, y}^{\alpha} u_{y} \cdot \xi^{y}+\frac{d}{d x}{ }^{C} D_{c, y}^{\alpha} u \cdot \xi^{x} \\
& \left.+\frac{1}{\Gamma(1-\alpha)} \frac{u_{y}(x, c)}{(y-c)^{\alpha}} \cdot \xi^{y}\right)+F \\
& \cdot \operatorname{div}(\bar{X})] d x d y=\iint_{D}\left[\left(\partial_{1} F\right.\right. \\
& \left.+\frac{1}{\Gamma(1-\alpha)} \frac{u_{y}(x, c)}{(y-c)^{\alpha}} \cdot \partial_{5} F\right) \xi^{y}+\left(\partial_{3} F \cdot \xi^{u}\right. \\
& +\frac{d}{d x}{ }^{C} D_{c, y}^{\alpha} u \cdot \partial_{5} F+\partial_{4} F \cdot D_{a, x}^{\alpha} u_{x} \\
& \left.+\frac{1}{\Gamma(1-\alpha)} \frac{u_{x}(a, y)}{(x-a)^{\alpha}} \cdot \partial_{4} F\right) \xi^{x}+\left(\partial_{2} F\right.
\end{aligned}
$$

$$
\begin{aligned}
& \left.\left.\cdot I_{x, b}^{1-\alpha} \partial_{4} F\right)+\frac{\partial}{\partial y}\left(\delta u \cdot I_{y, d}^{1-\alpha} \partial_{5} F\right)\right) d x d y \\
& =\iint_{D}\left[\left(\partial_{1} F+\frac{d}{d x}{ }^{C} D_{c, y}^{\alpha} u \cdot \partial_{5} F+\partial_{4} F \cdot D_{a, x}^{\alpha} u_{x}\right.\right. \\
& \left.+\frac{1}{\Gamma(1-\alpha)} \frac{u_{x}(a, y)}{(x-a)^{\alpha}} \cdot \partial_{4} F\right) \xi^{x}+\left(\partial_{2} F\right. \\
& +\frac{d}{d y} D_{a, x}^{\alpha} u \cdot \partial_{4} F+\partial_{5} F \cdot D_{c, y}^{\alpha} u_{y} \\
& \left.+\frac{1}{\Gamma(1-\alpha)} \frac{u_{y}(x, c)}{(y-c)^{\alpha}} \cdot \partial_{5} F\right) \xi^{y}+\left(\partial_{3} F \cdot \xi^{u}\right. \\
& \left.-D_{x, b}^{\alpha} \partial_{4} F \cdot \delta u-D_{y, d}^{\alpha} \partial_{5} F \cdot \delta u\right)+F \\
& \cdot \operatorname{div}(\bar{X})] d x d y+\oint_{\partial D}(-\delta u
\end{aligned}
$$$$
\left.\cdot I_{y, d}^{1-\alpha} \partial_{5} F\right) d x+\left(\delta u \cdot I_{x, b}^{1-\alpha} \partial_{4} F\right) d y
$$$$
=\iint_{D}\left[\left(\partial_{1} F+\frac{d}{d x}{ }^{C} D_{c, y}^{\alpha} u \cdot \partial_{5} F+\partial_{4} F \cdot D_{a, x}^{\alpha} u_{x}\right.\right.
$$$$
+u_{x} \cdot D_{x, b}^{\alpha} \partial_{4} F+u_{x} \cdot D_{y, d}^{\alpha} \partial_{5} F
$$$$
\left.+\frac{1}{\Gamma(1-\alpha)} \frac{u_{x}(a, y)}{(x-a)^{\alpha}} \cdot \partial_{4} F\right) \xi^{x}+\left(\partial_{2} F\right.
$$$$
+\frac{d}{d y}{ }^{C} D_{a, x}^{\alpha} u \cdot \partial_{4} F+\partial_{5} F \cdot D_{c, y}^{\alpha} u_{y}+u_{y} \cdot D_{x, b}^{\alpha} \partial_{4} F
$$$$
\left.+u_{y} \cdot D_{y, d}^{\alpha} \partial_{5} F+\frac{1}{\Gamma(1-\alpha)} \frac{u_{y}(x, c)}{(y-c)^{\alpha}} \cdot \partial_{5} F\right) \xi^{y}
$$$$
+\left(\partial_{3} F-D_{x, b}^{\alpha} \partial_{4} F-D_{y, d}^{\alpha} \partial_{5} F\right) \xi^{u}+F
$$$$
\cdot \operatorname{div}(\bar{X})] d x d y+\oint_{\partial D}(-\delta u
$$$$
\left.\cdot I_{y, d}^{1-\alpha} \partial_{5} F\right) d x+\left(\delta u \cdot I_{x, b}^{1-\alpha} \partial_{4} F\right) d y
$$

Definition 19. If the fractional action is invariant under the infinitesimal transformations (74) of group, i.e., for each of the infinitesimal transformations, the formula

$$
\Delta J=0
$$

holds, then the infinitesimal transformations are called the symmetric transformations in sense of Noether for the fractional system.

By Definition 19 and (73) and (85), we can get the following theorem. 
Theorem 20. For the infinitesimal transformations (74) of group, if the condition

$$
\begin{aligned}
& \left(\partial_{1} F+\frac{d}{d x}{ }^{C} D_{c, y}^{\alpha} u \cdot \partial_{5} F+\partial_{4} F \cdot D_{a, x}^{\alpha} u_{x}+u_{x}\right. \\
& \cdot D_{x, b}^{\alpha} \partial_{4} F+u_{x} \cdot D_{y, d}^{\alpha} \partial_{5} F+\frac{1}{\Gamma(1-\alpha)} \frac{u_{x}(a, y)}{(x-a)^{\alpha}} \\
& \left.\cdot \partial_{4} F\right) \xi^{x}+\left(\partial_{2} F+\frac{d}{d y} C_{a, x}^{\alpha} u \cdot \partial_{4} F+\partial_{5} F \cdot D_{c, y}^{\alpha} u_{y}\right. \\
& +u_{y} \cdot D_{x, b}^{\alpha} \partial_{4} F+u_{y} \cdot D_{y, d}^{\alpha} \partial_{5} F \\
& \left.+\frac{1}{\Gamma(1-\alpha)} \frac{u_{y}(x, c)}{(y-c)^{\alpha}} \cdot \partial_{5} F\right) \xi^{y}+\left(\partial_{3} F-D_{x, b}^{\alpha} \partial_{4} F\right. \\
& \left.-D_{y, d}^{\alpha} \partial_{5} F\right) \xi^{u}+F \cdot \operatorname{div}(\bar{X})=0
\end{aligned}
$$

is satisfied, then the transformations (74) are the Noether symmetric transformations for the dynamical system based on the action.

\section{Examples}

Example 21. Consider the functional

$$
\begin{aligned}
& J(u)=\iint_{D}\left(\left({ }^{C} D_{0, x}^{\alpha} u(x, y)-\frac{x^{1-\alpha}}{\Gamma(2-\alpha)}\right)^{2}\right. \\
& +\left({ }^{C} D_{0, y}^{\alpha} u(x, y)-\frac{y^{1-\alpha}}{\Gamma(2-\alpha)}\right)^{2}+x^{2} \\
& \left.+y^{2}\right) d x d y
\end{aligned}
$$

subject to the restriction $u(x, 0)=0, u(0, y)=y$ and $D=$ $[0, b] \times[0, d]$. From Theorem 11 , the necessary condition that every minimizer of the functional must fulfill is the following:

$$
\begin{aligned}
D_{0, x}^{\alpha} 2 & \left({ }^{C} D_{0, x}^{\alpha} u(x, y)-\frac{x^{1-\alpha}}{\Gamma(2-\alpha)}\right) \\
+ & D_{0, y}^{\alpha} 2\left({ }^{C} D_{0, y}^{\alpha} u(x, y)-\frac{y^{1-\alpha}}{\Gamma(2-\alpha)}\right)=0
\end{aligned}
$$

We see that

$$
u^{*}=x+y
$$

satisfies these conditions. Also, using the Legendre condition, a local minimizer for the functional must verify (26) and (27), which for our example is verified. In fact, $\partial_{44} F\left[u^{*}\right]=2>0$ and $\partial_{44} F\left[u^{*}\right]=2>0$.
Example 22. Let

$$
\begin{aligned}
& J(u)=\iint_{D}\left(\left({ }^{C} D_{0, x}^{\alpha} u(x, y)\right)^{2}+\left(\frac{x^{1-\alpha}}{\Gamma(2-\alpha)}\right)^{2}\right. \\
& +\left({ }^{C} D_{0, y}^{\alpha} u(x, y)-\frac{y^{1-\alpha}}{\Gamma(2-\alpha)}\right)^{2}+x^{2} \\
& \left.+y^{2}\right) d x d y
\end{aligned}
$$

subject to the restrictions $u(x, 0)=0, u(0, y)=y$ and

$$
\iint_{D}\left({ }^{C} D_{0, x}^{\alpha} u(x, y) \cdot \frac{x^{1-\alpha}}{\Gamma(2-\alpha)}\right) d x d y=c
$$

where $D=[0, b] \times[0, d]$ and $c=\iint_{D}\left(x^{1-\alpha} / \Gamma(2-\alpha)\right)^{2} d x d y=$ $1 / \Gamma(3-\alpha)$.

The function

$$
u^{*}=x+y
$$

satisfies the necessary conditions, as stated in Theorem 15.

Example 23. Let us consider the following fractional problem of the calculus of variations:

$$
\begin{gathered}
J(u)=\iint_{D}\left(u(x, y)\left(D_{x, b}^{\alpha} 1+D_{y, d}^{\alpha} 1\right)\right. \\
\left.+{ }^{C} D_{a, x}^{\alpha} u(x, y)+{ }^{C} D_{c, y}^{\alpha} u(x, y)\right) d x d y
\end{gathered}
$$

subject to the restriction $u(x, c)=0, u(a, y)=y$ and $D=$ $[a, b] \times[c, d]$.

If the transformation is $\left(\xi^{x}, \xi^{y}, \xi^{u}\right)=(0,0, c)$, where $c$ is arbitrary constant, then the fractional integral functional is invariant under Definition 19. In fact, the transformation satisfies (87).

\section{Data Availability}

No data were used to support this study.

\section{Conflicts of Interest}

The authors declare that they have no conflicts of interest.

\section{Acknowledgments}

This research is supported by the Doctoral Fund of Education Ministry of China (20134219120003), the Natural Science Foundation (61473338), and Hubei Province Key Laboratory of Systems Science in Metallurgical Process (Y201705).

\section{References}

[1] F. Riewe, "Nonconservative Lagrangian and Hamiltonian mechanics," Physical Review E: Statistical, Nonlinear, and Soft Matter Physics, vol. 53, no. 2, pp. 1890-1899, 1996. 
[2] R. Almeida, "Optimality conditions for fractional variational problems with free terminal time," Discrete and Continuous Dynamical Systems - Series S, vol. 11, no. 1, pp. 1-19, 2018.

[3] R. Almeida, "Variational problems involving a Caputo-type fractional derivative," Journal of Optimization Theory and Applications, vol. 174, no. 1, pp. 276-294, 2017.

[4] J. Zhang, X. Ma, and L. Li, "Optimality conditions for fractional variational problems with Caputo-Fabrizio fractional derivatives," Advances in Difference Equations, Paper No. 357, 14 pages, 2017.

[5] R. Almeida, S. Pooseh, and D. F. Torres, "Fractional variational problems depending on indefinite integrals," Nonlinear Analysis. Theory, Methods \& Applications. An International Multidisciplinary Journal, vol. 75, no. 3, pp. 1009-1025, 2012.

[6] A. A. Kilbas, H. M. Srivastava, and J. J. Trujillo, Theory and Applications of Fractional Differential Equations, New York, NY, USA, Elsevier, 2006.

[7] K. S. Miller and B. Ross, An Introduction to the Fractional Calculus and Differential Equations, John Wiley, New York, NY, USA, 1993.

[8] S. G. Samko, A. A. Kilbas, and O. I. Marichev, Fractional Integrals and Derivatives, Theory and Applications, Gordon and Breach, Yverdon, Switzerland, 1993.

[9] S. Abbas, M. Benchohra, and A. N. Vityuk, "On fractional order derivatives and Darboux problem for implicit differential equations," Fractional Calculus and Applied Analysis An International Journal for Theory and Applications, vol. 15, no. 2, pp. 168-182, 2012.

[10] D. Tavares, R. Almeida, and D. F. Torres, "Constrained fractional variational problems of variable order," IEEE/CAA Journal of Automatica Sinica, vol. 4, no. 1, pp. 80-88, 2017.

[11] E. Noether, "Invariant variation problems," Transport Theory and Statistical Physics, vol. 1, no. 3, pp. 186-207, 1971.

[12] G. S. Frederico and M. J. Lazo, "Fractional Noether's theorem with classical and Caputo derivatives: constants of motion for non-conservative systems," Nonlinear Dynamics, vol. 85, no. 2, pp. 839-851, 2016.

[13] Y. Zhang and X.-S. Zhou, "Noether theorem and its inverse for nonlinear dynamical systems with nonstandard Lagrangians," Nonlinear Dynamics, vol. 84, no. 4, pp. 1867-1876, 2016.

[14] T. M. Atanacković, S. Konjik, S. Pilipović, and S. Simić, "Variational problems with fractional derivatives: invariance conditions and Nöther's theorem," Nonlinear Analysis: Theory, Methods \& Applications, vol. 71, no. 5-6, pp. 1504-1517, 2009.

[15] B. Yan and Y. Zhang, "Noether's theorem for fractional Birkhoffian systems of variable order," Acta Mechanica, vol. 227, no. 9, pp. 2439-2449, 2016.

[16] D. Z. Lao, Fundamentals of the Calculus of Variations, vol. 29, National Defense Industry Press, China, 3rd edition, 2016.

[17] M. J. Lazo and D. F. Torres, "The Legendre condition of the fractional calculus of variations," Optimization. A Journal of Mathematical Programming and Operations Research, vol. 63, no. 8, pp. 1157-1165, 2014. 


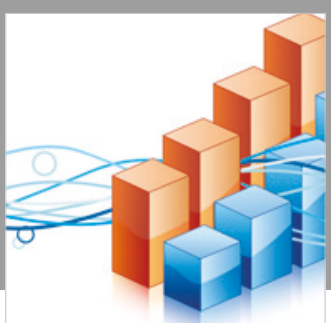

Advances in

Operations Research

\section{-n-m}
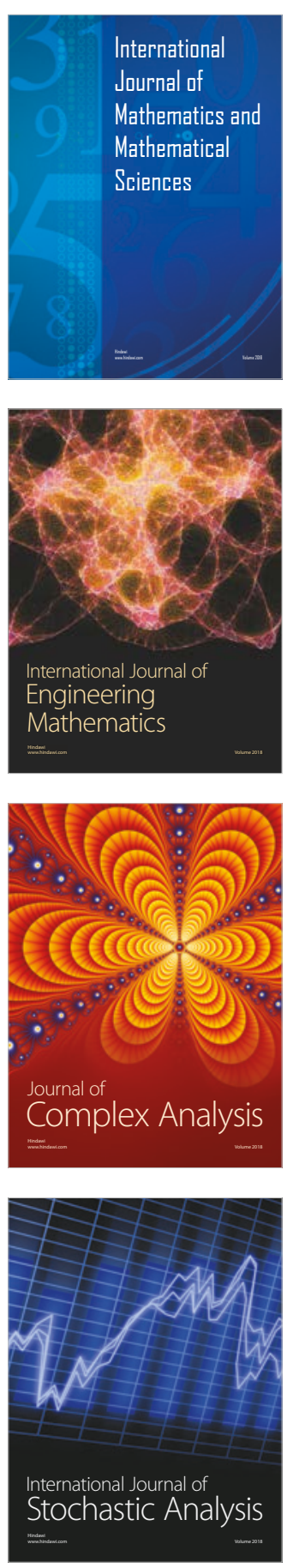
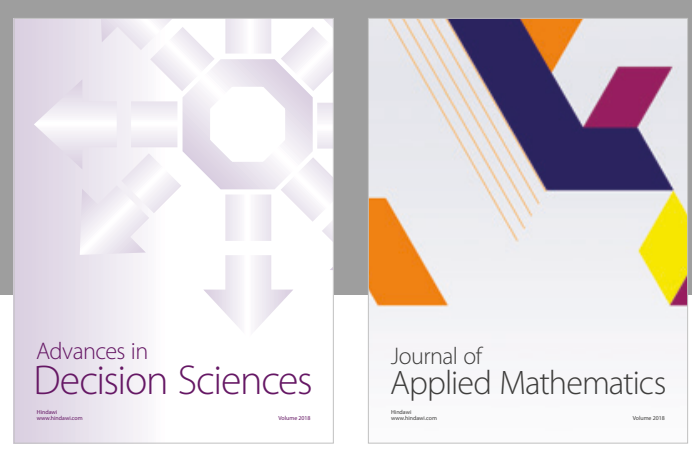

Journal of

Applied Mathematics
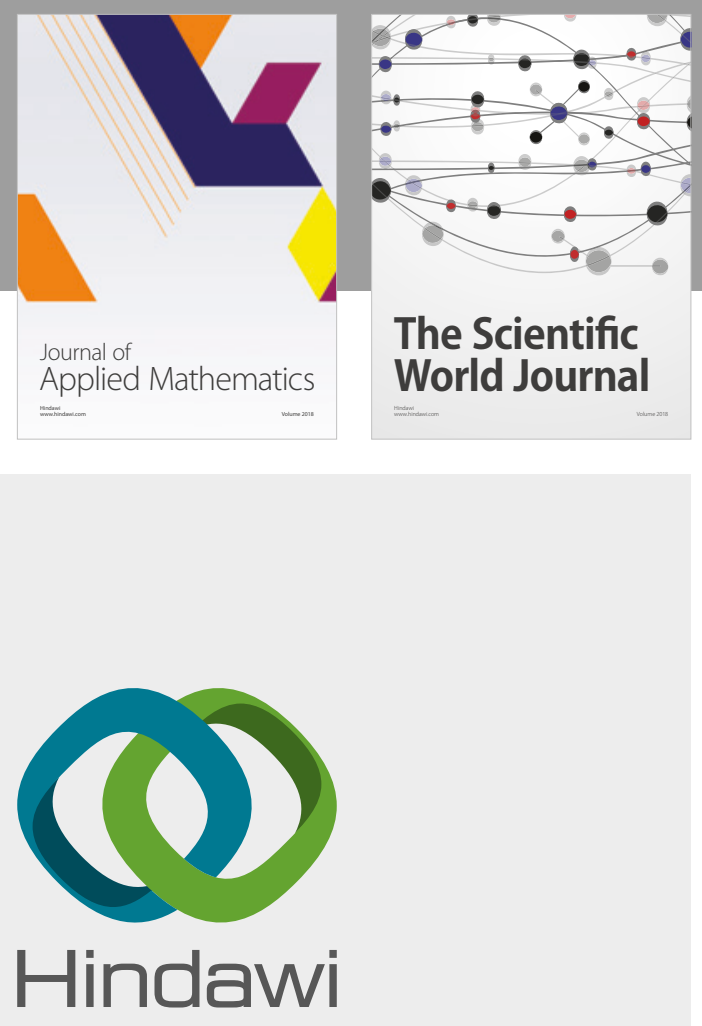

Submit your manuscripts at

www.hindawi.com

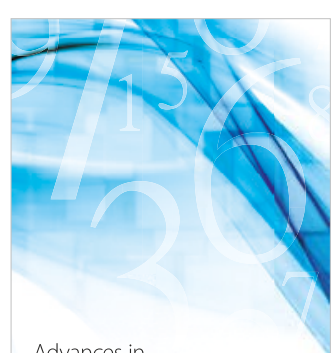

Advances in
Numerical Analysis
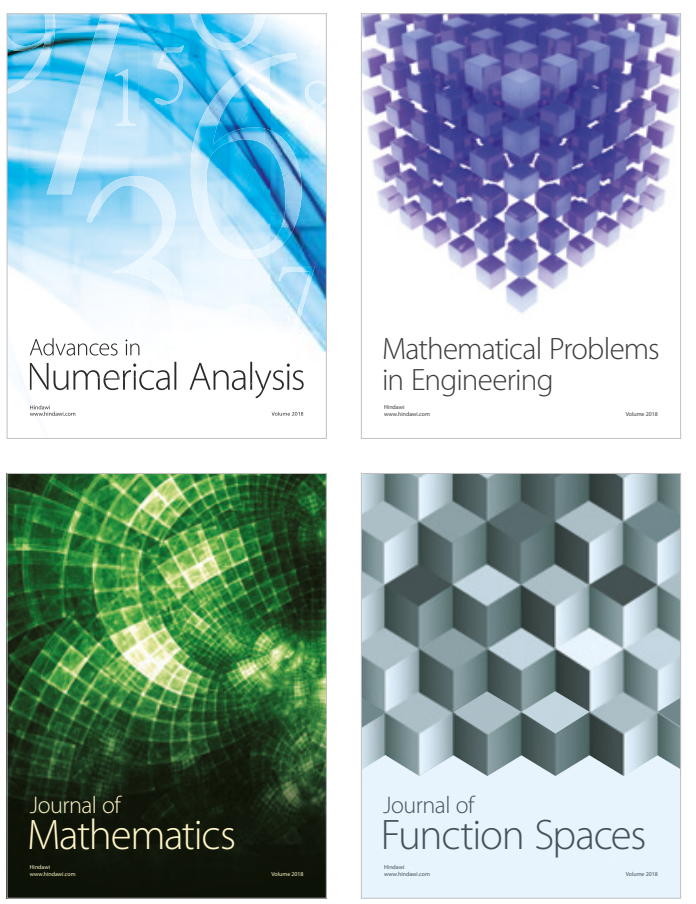

Mathematical Problems in Engineering

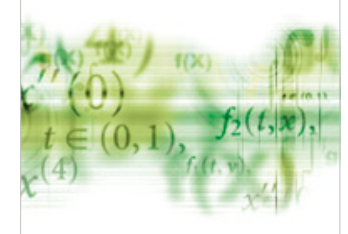

International Journal of

Differential Equations

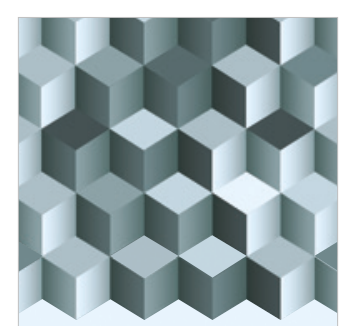

Journal of

Function Spaces

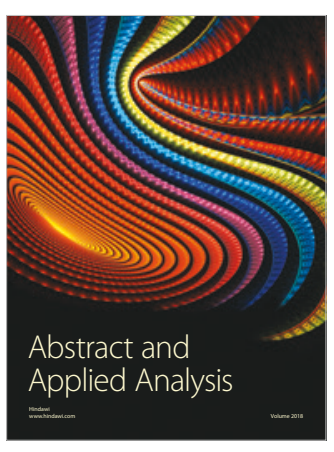

The Scientific

World Journal

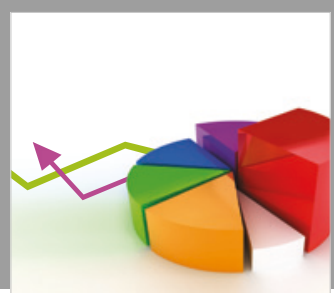

Journal of

Probability and Statistics
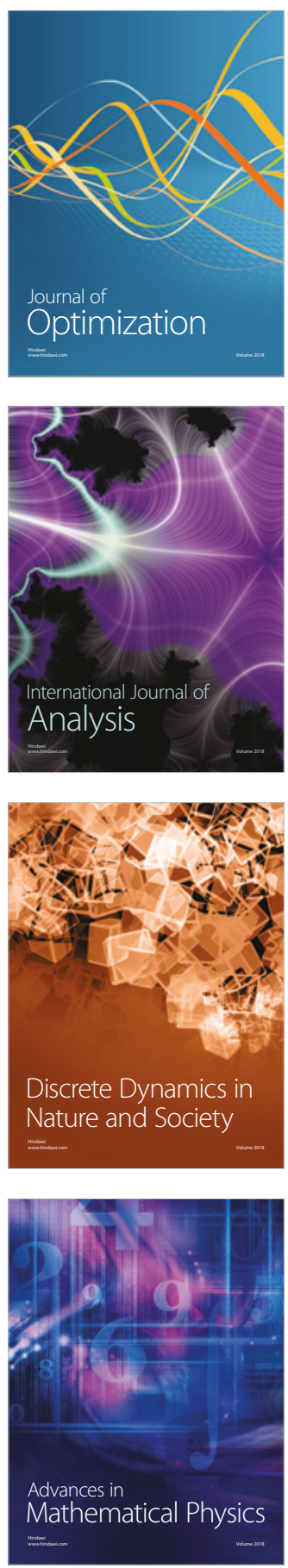